\title{
FLEXIONSVERHÄLTNISSE BEI ROBERT VON GLOUCESTER.
}

\author{
[Fortsetzung.]
}

69. B. Plural.

Nóminativ, accusativ, vocativ.

1) Die normale endung ist -es, an wörter auf tonloses $e$ tritt naturlich nur $s$ an. Belege für die gruppe a: $\alpha$ ) ae. masculina: godes 694, peues 11293 (sg. pef 5621), clopes 2306, stones 3067 , erles, kniztes 3115 , clerkes 9529 , gostes 7510 , wolues 5299 , gottes 9162 , walles 422 , cumelinges 424 , ssillinges 8933 , abhodes 10172 , louerdes 752 , hauekes 5571 , monekes 4743 , u. s. w. B) ae. neutra: ssipes 1739. 6018, bapes 3074 , leues blätter 1288 , wines 755 , bōnes 4592 , tounes 1390 , eres ähren 10045, londes 2227 , nordes 2918 , suerdes 4448 , riztes 7342.10724 , pinges 3052 , bernes 7152 , cornes 4193 .

Anm. 1. Würter anf -er, -el: Zı taper gehört taperes 11022, zu water vateres 2115. 2800, zu wonder wondres 151; maister schwankt zwischen maistres 35.391 ö. und maisters $928.7330 \ddot{0}$. - mantel hat als plural mantles 11176 ( vgl. 11174), iiber castles s. $§ 98$ anm. 2. - Vgl. noch of herdles 4785 (ae. hyrdel).

Ann. 2. bissop hängt oft nur $s$ an: bissops 10165 ö. neben bissopes 7891 ï. (vgl. auch of sterlings [d?] 11840 neben of sterlinges 11878, und of kinedoms 4366).

Anm. 3. Der alte plural Wealas ${ }^{1}$ ist in seiner erstarrten form Walis zin einem singular geworden. Einmal findet man Wales geschrieben (41), aber im reime zu ivis, und sonst hat die endung stets ein $i$ : al $W$ alis (a)

I Ich setze dieses wort nach meiner 2. these (vgl. die vorletzte seite der diss.) mit $e a$, nicht $\hat{e} a$ (Sievers $e ́ a$ ) an. Zu einer ausfihrlichen erörterung der frage ist hier nicht der ort, doch will ich wenigstens das eine erwähnen, dass ich in den von Sievers (Paul u. Braune's Beiträge X) zum beweise seiner hypothese beigebrachten versen statt $r, l: r h$, lh einfiihren will (wegen der berechtigung einer solchen ïnderung vgl. Sievers, Ags. Gr. $\$ 218$, anm. 3). Auf diese weise werden die hebungen an den betreffenden stellen einfach durch position lang gemacht, die verse beweisen also nichts für Sievers' regel. (NB. Auf seite 32, zeile 2 v. o. meiner diss. ist im widerspruch zu der these irrtiumlich sweora statt sweora stehen geblieben).

Anglia. N. F. I. 
109. $543 \mathrm{R}$, of, in, to Walis (d?) 85.5126 (: ywis). 7668 (: his eius). 10768 (: amis). Ueber Hailes s. diss. $\S 8$.

Aus der gruppe b begegnet ein plural bowes äste (n) 5748, aus c trifft man weies 169.9313, aus d bodies 6219. 6544.8139, aus e pies 8633. Der plural zu dai heisst in $\mathrm{A}$ immer daves = ae.dagas (B hat gewöhnlich dayes, kennt aber auch dawes) 5382. 6670. 7167, und zu peni gehört als mehrzahl panes 1392*. 9720*† (vgl. die formen panenes, ponewas im Laz. B).

Anm. 4. Auch der alte plural Norpwegas ist zu einem singular geworden, aber ohne, wie $W$ alis, das $s$ zu bewahren: Norpwey (n) 6311, (a) 3784, of $N$. 2155. 3767. 3905, Norpweye (a) 4616, of N. 2253 R. 3787.

$\mathrm{Zu}$ lime (h 2) gehört als plural limes 186. 6574. 11729, lumes 3595, zu zal(e) zales 1023. 6120, zu maide maidens 2101. 2105 ö., maidenes (nur 11897).

2) Mehrere ursprïngliche neutra haben einen endungslosen plural: hous 1176. 8579, hors 2052. 8337.8466, ssep 2202. 7211, pound 5568. 5949.

Anm. 5. zer bildet zeres 5035. 5282. 6669 und zer 1386. 7640 ö. (: er adv.); zeres steht nicht mit vorangehendem, wol aber mit nachfolgendem bestimmtem zahlwort (vgl. Morsbach l. c. p. 113). - heued schwankt zwischen heued 179i*. 7449 und heueden 2378*. 5254. 8293 (nach B).

3) Vereinzelt begegnet als endung -en: deuelen 10417, applen 5747. 5756, heueden (s. anm. 5).

Anm. 6. Bei dores tore 10198, doren türen 10189 kann man wegen der doppeldeutigkeit des $o$ nicht entscheiden, ob ae. dor oder duru zu grunde liegt.

Anm. 7. Der plural zu ssọ lautet ssọ̄n 65 78.

8 70. Dativ pluralis.

Ganz vereinzelt bêgegnen einige dative pl. anf $-e$, welche direct von den ae. formen anf -um herzuleiten sind: of kinge 8179 R, of pinge 703 R. 6079 R, of house 5495 , of dawe 5907 R. 6362 R. $8327 \mathrm{R}$, of lifdawe 1234 R. 3151 R. $6695 \mathrm{R}$ ö. Sonst stimmt der dat. mit dem nom. uberein: clerkes 3975, kniztes 3976, erles 10744, of kinges 1633, of wolues 5774 , of walles 8494 , of monekes 8921, of foweles 1212 , of maisters 8293 neben to maistres 4449, mid taperes 11026, of erchebissops 3933 neben of (erche-) bissopes 6762. 8390, of neizebores 11143. 11145, in ssipes 912 . of tounes 5427, of, in londes 421. 4376, of pinges 6324 R. 3046 ö., of riztes 1089 , of wordes 860 , of wondres 162 , of panes 1397 , of holes 165, of zates 8113, of limes 8497. 9755, of maydens 2116. - of zeres 5041, of zer 241 (: er adv.). 3477. 5881 ö., mid, of hors 1281. 3913, to hous 5564 . 
Anm. 1. Neben poru alle pinge 2271 (: to kinge). 10864 (: aze kinge) steht häufigeres por(u) alle ping 1870. 66.11. 10018. 10971 r. a. king nsg.; auch das por alle pinge $6 S 1$ ist wegen des reimes zu king nsg. in p.a. ping zu ändern. Ausserdem findet man noch mest of alle ping 2323 (: king nsg.). Wo pinge geschrieben ist, liegt natiirlich der alte dpl. pingum zu grunde, ob dagegen ping daraus verkürzt ist oder nicht vielmehr auf den alten endungslosen napl. ping zuriickgeht, bez. selbst acc. ist, dürfte schwer zil entscheiden sein. Doch bleibt zu beachten, dass der plural von ping sonst immer pinges lautet. - Dass ping $(e)$ in den genannten verbindungen singular sei, darf man wol kaum annehmen.

Anm. 2. Eine sehr starke verkürzung zeigt der dpl. in lende ( $=$ ae. léndenum, von lendenu neutr. pl.) 7732 (: bende inf.).

Anm. 3. In ausdriicken wie bi hor daye 1629, bi pre kinges daye 7285 , bi is daye $5696 \mathrm{R}$, bi pe kinges daye Edward 7287, bi pe kinges day Vortiger 4515 müchte man zunächst geneigt sein, das daie, dai als dpl. aufzufassen. Da dieser casus von dai aber sonst stets dave heisst, so muss dai(e) hier sg. sein, es bedeutet 'lebenszeit' (s. Engl. Stud. IV, 502).

$\$$ 71. Genitiv pluralis.

Direct auf ae. londa, pinga, horsa gehen londe 189, pinge 6737 (daneben 1565 alle fing mest), horse 10522 zurick. Vereinzelt trifft man die der schwachen declination entlehnte endung -en $(e)=$ ae. ena: clerken $(e)$ 7512*. 10206, monekene (C: $a$ monacus) 11478. Sonst geht der gen. pl. anf -es aus: kinges 3936 . 7285. 8746, knizles 1332, Scottes 994, londes 7246, bernes 10198.

\section{A e. $j o-s t a ̈ m m e$.}

(Masculina und neutra.)

\section{\$72. A. Singular.}

Fur den nom., acc., voc. sind folgende typen zu unterscheiden:

a) Wörter mit consonantischem auslaut (ae. paradigmen secg, cyn[n]): $\operatorname{rug}(h)$ (a) $9036^{*}$. 10795*, hul (n) 3623. 4223, (a) 1306. 3593, kun (n) 992.6774, (a) 309 .

b) Das wort hei (ae. hêg; kommt nur nach of 8399 vor),

c) Das wort glẹ (ae. glêo neben glìg; begegnet nur nach uor 5515).

d) Wörter auf tonloses $e$ : 1) nach den ae. paradigmen ende, fiscere, rîce: ende (n) $3516 \mathrm{R}$, (a) 2649. 7024 R. (an end lond $3699^{*}$ ), harpare (n) 5509, bissopriche (n) 5736 ö., (a) 6846. 8722 ï. (bissoprich acc. 117). - 2) nach dem ae. paradigma wêsten, mit verlust des $n$ : eue (ae. $\hat{e} f e n$ ) (begegnet nur mit azen, an 330 R. 10180 R; einmal findet man an Hal pores Euen 8120).

Im dativ sing. haben die wörter des typas a facultativ 
noch das alte flexivische $e$, vor welchem nattirlich die urspringliche gemination des stammauslautes noch erhalten ist: of, at rugge 9959 (pl.?). 7740, in hulle $5985 \mathrm{R}$ neben of, vpe hul 8211*. 6171.11520 , out of putte $2789 *$, to putte 11203 , to wedde 8083 , to bedde 7788. 8950, a childbedde 7789 neben to, in bed 7177 . [99]. 11654, of kunne 5092 R. 4896. 8889 neben fram, to kun 306. 930.

Anm. 1. In dem satze pat zo wolde euere eft in monnes bedde come 5433 ist bedde keinesfalls acc. Nach come scheint in, obschon es nach unserem gefühl auf die frage 'wohin' antwortet, den dat. regieren zu können. Man findet z. b. noch hii come per in pe felde 11855. Vgl. anch das oben erwähnte he dude him in pe weie.

Flexionslos ist der angefuhrte mutmassliche dat. von hei; bei gle hat, falls jenes uor gle 5515 ein dat. ist, eine angleichung dieses casus an den nom. stattgefunden (vgl. Sievers \& 247, anm. 3). - Bei den wörtern unter d 1 unterscheidet sich der dat. nicht vom nom. (Ob an, azen eue dative oder accusative sind, lässt sich nicht sagen.)

\section{B. Plural.}

Im nom. und acc. tritt -es an die wörter des typus a, $s$ an die des typus $d$. Die bildungen auf -ere unterdricken gelegentlich das $e$ vor dem $s$. Belege: rugges (a) 9438, puttes (n) 8465, briddes (n) 3673 (s. Stratmann, Engl. Stud. III, 357) endes (n) 1672 , leches (n) 7817. 9115, ssephurdes (n) 5359 , vissares (n) 5359, manquellers (n) 9333 (B: -ares), wolmongers (a) 11173 bissopriches (n) 126, (a) 3975 ö. - to hulles (dat.?) 2966. haben -es.

Anm. 2. Der npl. ssetare 7482 ist verschrieben, die anderen hss.

Anm. 3. In of ven $146 R$ könnte man einen endungslosen plur. vermuten, doch ist das wort wol singularisch gemeint und bedeutet 'sumpfland'.

Gen. pl.: bowiares 11222.

III. A e. wo-stä m me.

(Masculina und neutra.)

8 73. A. Singular. Die vorkommenden wörter sind: a) mit erhaltung, bez. vocalisation des $w$ : ae. $s n d w$, (Tîw): nom. snow, snou 1002. 9512 - dat. snowe $9514 \mathrm{R}$, (gen. Tiwes-day 11676 o.). b) mit verlust des $n$ : ae. $\operatorname{tr} \hat{e} o(w)$, $c n \hat{e} o(w)$ : nom. tre 7228 ö., acc. tre 5745 - dat. of tre $4401.8436 \mathrm{R}$, in kne 4961 R. - B. Plural. Ein plur. findet sich nur von tre, und zwar mit der endung $n$ : tren (a) 3544, of tren 11 (: sen inf. sehen). 1288, among tren 3542 . 
IV. A e. $\hat{a}$-s tämme.

\section{\$7. A. Singular.}

Wir unterscheiden zunächst folgende zwei haupttypen: a) kurzsilbige von ursprünglich 2 silben (ae.paradigma giefu), b) langsilbige von ursprtinglich 2 silben (ae. paradigma $\hat{a r}$ ). Die endung des nom. acc. und dat. ist in diesen beiden typen -e. Die wörter unter b haben dieses - $e$ im nom. angenommen, weil die anderen casus dasselbe hatten, und vielleicht auch nach dem beispiel der wörter unter a.

Belege: Nom. (typus a:) loue (ae. lufu st. oder lufe sw.) 1719 R. 5822, lawe 5277. 5813, fare 1202. 4302, ssame 3405. $8886 \mathrm{R}$; - (typus b:) leue (ae. lêaf) 11552, bọte [20], wę̄de (ae. $w \hat{c} d$ fem. oder gew $\hat{e} d e$ neutr.) $11735 \mathrm{R}$, mẹde 1255. 6385, rọ̄de 8634, liflode 833, spẹ̣che 2672 , wręche 4830 R. 8661, ssire 11071, horsbēre 3400 , sorwe 779. 7324, wombe 1556.6285 ö.; reste 624.0 , warde 11424 , sonde schickung 6822 , ssonde 1493 , wolle 7212 . Acc. (typus a:) loue $9403 \mathrm{R}$, note (ae. notu) $10008 \mathrm{R}$, lawe 10495 R. $10638 \mathrm{R}$, vnlawe $9705 \mathrm{R} \dagger$, care $9385 \mathrm{R}$, chaffare 11173, ansuere (ae. undswaru?) 1340 R. 4061, tale $3066 \mathrm{R}$, ssame $500 \mathrm{R}$; (typus b:) Frye npr. $4697 \mathrm{R}$, raketeie (ae. race[n]têah st. oder racentêage sw.) 3001, leue (ae. lêaf) $5002.10182 \mathrm{R}$, bọte $3503 \mathrm{R}$, mệde 1762, rộde 18622, liflode 974, spẹche 7538, wręche $5936 \mathrm{R}$, Chestressire 95, ōre $1312 \mathrm{R} .6981 \mathrm{R}$, lōre 5111, myle $3666 \mathrm{R}$, wile 1892, bẹne (ae. bêean) $10223 \mathrm{R}$, sorwe 5448. 8341, wombe 5871. 7443, reste 2971 (: hor beste, superl.), warde 8301, sonde $1835 \mathrm{R} .3727 \mathrm{R}$, slounde $2091 \mathrm{R} .10873 \mathrm{R}$, wounde $5305 \mathrm{R}$, halle 7865, wolle 10033. - Dat.: Abgesehen von dativen der obigen substantiva, findet man fur diesen casus noch folgende (mutmassliche) belege: (typ. a:) of, in wouke 2431. 4700; (typ. b:) of hide (hufe landes) 8933, in prowe (von ae.prag) $5252 \mathrm{R} .7813 \mathrm{R}$, in pine (schmerz, ae. * ${ }^{*} \hat{\imath} n$ ist zufällig nicht belegt) $9896 \mathrm{R}$, in cheste 1162, in fizle $2585 \mathrm{R}$, mid, wis arwe (pfeil) 5037. 8196. 8653, to nelde (nadel) 2199*.

Anm. 1. Neben in, of wombe 6927. 7073. 7791 ö. steht einmal 5681 in womb, und zwar im reim zu einem unzweifelhaften dat. lomb. Da auch der nom. und acc., wie wir sahen stets nombe lautet, hat man wol ein recht, an jener stelle mit $\alpha$ lombe, wombe zu schreiben. Vgl. ubrigens auch den reim 76199 .

Anm. 2. Wie das dem ae. of dìne entsprechende adverb teils adoune, teils adoun lautet (s. $\$ 132,6)$, so begegnet auch vom subst. doune (n) 7481 , vgl. 3620, eine endungslose form (vp..) doun (a?) 11364 . 
Anm. 3. Das ae. foreweard hat vorewarde ergeben (n) 6323. 6415. 7140. 7348, (a) 7134. 7340. 8038, doch findet man ohne $e$ : voreward (n) 4305 , of foreward (d?) 5007 .

Anm. 4. Zum nom. stret 172. 143. $174 \mathrm{R}$ (Erninge, Ykenilde, Watelinge stret) gehören als (vermutliche) dative in, fram stret $154 \mathrm{R} .6074$ und amidde strete $1814 \mathrm{R}$, fram strete to strele $6392 \mathrm{R}$; zu help (n) 2206. 7933, (a) 1961. 3637 of help 4331. [146] 8. und of helpe 11099; zu March Mercien (a) 5134. 5476 of March 60. 90. 91 ö. und of Marche [134]; zu Denemarch (a) 3785 of Denemarch 52. 909 ö. und to Denemarche 912. - flọd hat im acc. kein -e (8594 R), dagegen heisst es mid flode 473 (: mid alle gode), in, of flode $1123 \mathrm{R} .6561 \mathrm{R}$.

Anm. 5. Nie mit -e erscheinen, vielleicht nur zufällig, half hälfte, seite (n) 8270, (a) 92, (d ?) in $h .1200 .1279$ ö., marc mark geldes (a) 10460, (d?) of $m .8019$, march landesmark (s. diss. $§ 79$, anm. 5) (n) 110, und uọn mittagsstunde: ouer $n$. (a?) $73112 \mathrm{R}$, vorto $n$. (d?) $8198 \mathrm{R}$.

Anm. 6. Von wörtern, deren stamm im ae. nom. sg. auf eine tonlose ableitungssilbe (mit ursprïnglichem oder secundärem vocal) ausging, sind sâvol, ceaster, eornest und weorold vertreten: soule (n.) 3604. 567t, (a) 1615. 1651, (d) 7314 - Chestre ortsn. (n) 149, Gloucestre (a) 66, Winchestere (a) 10546. (S. diss. § 151, anm. 4.) - ernest (a) 2682.8282 ö., (d ?) mid e. 6274. 6279. - world (n) 479.3997 u. sehr oft, (a) 2429 , (d ?) in $w$. 255. $1572 \mathrm{u}$. sehr oft (of worl 5116) neben vereinzeltem worlde $2162(\mathrm{n})$, in worlde ( $\mathrm{d}$ ?) 565 .

Anm. 7. Dazu kommen die verbalsubstantiva auf -ing, welche im nom. acc. dat. sg. das flexivische $-e$ beliebig anhängen oder weglassen. Ohne -e: nsg. 7706. 10142. 11185 (: king nsg.), asg. 2098. 5603. 5895 (: king nsg. asg.), dsg. (?) nach in, at 5029. 7845. 9577 (: king nsg.). - Mit - $e$ : nsg. 8150.10401 (: bringe inf.), asg. $2763.9695 \dagger .10386$ (: bringe inf.), nach fram, at, of 233. 2830. 5974 (: bringe inf.).

Anm. 8. Das abstracta bildende suffix ae. $-p u,-p$ erscheint bei Robert im nom. acc. dat. sg. stets als -pe: strengpe (n) 161. 8814, (a) 9574, (d?) of s. 7730 - treupe (n) 4985, (a) 3584, (d?) of tr. 500t - reupe (n) 6912, (a) 5000, (d ?) vor r. 756 - wrappe (a) 828, (d ?) in $w .763$ - murpe (n) 1156 , u. s. w.

875. Gen. sing.: Auf -e gehen aus, wie im Ae.: rode 1932. 6596, ssame 3460 ; world bildet worldes 199.6442 (worles 1073*. 1076*. 3762).

\$ 76. B. Plural.

Nom., acc. und voc. dieses numerus endigen

1) auf -e (entsprechend der ae. flexion): wouke 7263. 10099. 12010, mile 5705. 5845. $11150 \mathrm{R}$ ö.

2) auf - en (nach analogie der $n$-stämme): arwen pfeile 5306 (B: arewe). 8123, elnen ellen 8834, ssoldren 8267.

3) auf -es (nach analogie der starken msc.): lawes 1658. 2279 ö., stretes 11221, pynes 4189, sornes 2834.5935 ö. 
4) Sie sind endungslos bei dem subst. marc 'mark geldes' (wol nach dem beispiel von pound) 8084 (: starc adj.). 8769. 10026 und bei ssipuol 917. 4671. 536:5. 5925 ö.

Von (vermutlichen) dativen findet man dementsprechend mid arwen 1119, in ssoldren 7732, in halles 3972, mid ssipuol 2401.

Anm. Mehrere wörter bilden ihren plural bald auf die eine, bald auf die andere weise: hide (n) 6016, hiden (n) 7676; ssire (n) 1390 (B: schiren), ssiren (n) 5338 , (a) 63; soule (a) 4462 (B: soulys), soules (a) 11701 (vgl. 6857), vor soulen 7591 (B: -es). 6513; wounde (a) 11397, wounden (11) 4089. 4583, (a) [43], wip woundes (B: -en) 4412. - Neben bi halues 'bei seite' steht a four half Engelond 'nach allen vier teilen von E.' 10875 und a uour alf pis lond 'in allen vier teilen dieses landes' 5942.

Der einzige vorkommende gen. pl. ist soule $7670^{*}$.

\section{Ae. $j \hat{a}$-stämme.}

\$ 77. A. Singular. - Im nom., acc., voc. endigen

a) auf $-e$ :

1) Die ursprunglich kurzsilbigen nach dem muster des ae. gen. dat. acc. sg.: brugge (n) 8286. 11095, (a) 7316. $11256-$ Grantebrugge (a) 39. 71, in helle (a ?) 4283, sunne (n) 9041, (a) 6932. - Ausnahme: hen (a) 8335 (s. ten Brink, Chauc. § 207, 2).

2) Die abstracta auf -nes nach dem muster des ae. gen. dat. acc. sg.: (n) 6811. $8825 \mathrm{R}$, (a) 4031. $7236 \mathrm{R}$.

3) condel nach dem muster des ae. gen. dat. acc. sg., aber mit syncope des inlautenden $e$ nach vorausgehender vereinfachung des $l$ : candle (a) 10370. (Der nom. kommt nicht vor, wird aber kaum anders als candle gelautet haben.)

4) Einige wörter auf -en nach dem muster des ae. nom. sg., mit verlust des -n: żẹme (ae. gîemen, gêmen) (a) 2862. $4008 \mathrm{R}$, kunrede, kinrede (n) 9552. 5764, (a) 346.

b) auf consonanten:

Die ursprthnglich langsilbigen: zerd gerte (a) 5888, hind cerva (a) $7712 \mathrm{R}$, ax axt (a) 388. 573. - Ausnahme: milce (n) 8004, (a) 920.6981.

Die (mutmasslichen) dative entsprechen den nominativen: vpe, at brugge 7307. 8285, of Grantebrugge 6027, of, to helle 7213 R. 4462 R, of sunne 7193, in -nesse 3012 R. 9984 R. $10923 \mathrm{R}$, vor kunrede 9553 , vor sibrede 10108 , mid ax 1122.6060 , zn hind jedoch gehört mid hinde $8568 \mathrm{R}$.

Ein genitiv ist brugge 11180 .

Anm. Dem ae. blîps, bliss entspricht blis (n) $5686 \mathrm{R}$. $7264 \mathrm{R}$ (statt of ist dort and zu lesen), (a) $4079 \mathrm{R}$. $7226 \mathrm{R}$, (d ?) to, into, of blis $5651 \mathrm{R}$. 
5677 R. 6266 R. Flexivisches -e begegnet bei diesem worte nur in der formelhaften verbindung ioie \& blisse inou (n) 6952. 9620†. 11569 (nach in, mid, wip 3873. 9030. 11823). Ist blisse hier gen. sg., abhängig von inou? (vgl. Engl. Stud. VII, 140). - Auch die Brandanlegende p. 9 hat joye \& blisse ynouz.

8 78. B. Plural.

Als pluralendungen begegnen -e, -en und -es. Nom. und acc.: eluene 2754 (ae. ielfen = mhd. elbinne) - bruggen 11612, candlen 5893. 7795 - ribbes 518. - Dat.(?): of condlen 5889, of zerden 4788. 6985 - of, among hegges 2677. 4299, wit axes 11199. - sunne schwankt zwischen beiden flexionsweisen: sunnen (a) 5345 (B: -es), sunnes (a) 5920 (BC: -e), fram, of sunnes 5057 (B: -e). 10316. 10319; vielleicht ist anch vor sunne 5093. R plural, vgl. auch 3604 of sinne in A (synnys in B).

\section{Ae. $w \hat{a}$-stämme.}

\$ 79. Vertreten sind von den wörtern mit vocal vor dem $w$ ae. $\operatorname{trêon}$ (d. h. *ortrêow): dsg.(?) in ortrou (C: -owe) 7021, von denen mit consonant vor dem $w$ das ursprtinglich kurzsilbige ae. sceadu: nsg. ssade 2330 und die ursprtinglich langsilbigen $m \hat{e} d$ und $l \hat{e} s$. Diese beiden letzten erscheinen beim antreten einer endung stets ohne das $w$. Da sie sowol 'wiese' als auch 'wiesenland' bedeuten können (vgl. ven $\S 72 \mathrm{~B}$ ), ist bei manchen ihrer formen der numerus unklar. Unzweideutige singularformen sind to, into, out of med 9517. 11244. 11255. 11256 und sichere pluralbildungen medes (a) 10194, in medes (d?) 3963 und of lesen 15. Falls die lesart in A v. 1005: lese last per alle winter richtig ist $(\mathrm{B} \alpha \beta \gamma \delta$ haben lastep, $-i j$, was auch plural sein kann), so wäre durch diese stelle auch noch ein nsg. lese nachgewiesen. Dagegen lassen uns folgende sätze ganz im zweifel uber den numerus der darin vorkommenden lese und mede: 3887: in pe oper half bep grele wodes, lese \& mede also. - 7704: \& made it al forest \& lese. - $7700 \mathrm{f}$.: vor pulke (sc. pe Nine Forest) he louede inou \& astorede wel mid bestes \& lese. - 11-15: vor Engelonde is vol inoz... (of lesen) $\&$ of mede (r. a. of lede von blei).

VII. Ae. $i$-stämme.

80. 1. Masculina und neatra.

A. Singular. - Nom., acc. und voc. endigen

a) auf -e bei den ursprtinglich kurzsilbigen: slape (a) 6942, 
mete speise (n) $3672.9115 \mathrm{R}$, (a) 370 , bite (a) $4214 \mathrm{R}$, smite (n) 9013, ssute (a) $8132 *$. 11103* (C bat beidemale schote), stede, stude ort (n) 2185. 5306. 889. 1570, (a) 3223. 5874, biquide (a) 7826. 7887, spousbruche (a) 625 , eye schreck (a) $9617 \mathrm{R}$, lere (ae. lyre) (n) 10813, chele (n) 153, ssiprene (ae. ryne) (n) 341. Suftix -scipe: frendssipe (a) 818. 1083.

b) auf consonant bei den ursprüglich langsilbigen: dunt (n) 4228. 6283, (a) 400. 3837, stench (n) 8354, drench (a) 1555 ö., streng (n) 9353 , worm (n) 2811, (a) 1006* - fless (s. Sievers § 267) (a) 8447 ö.

Der dat. ist bei den substantiven nnter a gleich dem nom.: at mete $2511 \mathrm{R}$, in * stede $7866 \mathrm{R}$, vgl. auch noch in mone $5937 \mathrm{R}$. $8915 \mathrm{R}$ (ae. myne) und vnder spere $11861 \mathrm{R}$. - Die wörter unter b bilden ihn bisweilen durch anhängung von-e: vor stenche 8500 , of, mid wrenche 7441 R. 11053 R ö., of flesse 5815, lassen ihn aber auch oft ohne flexionszeichen: of, mid dunı 7477. 6280, of flizt 4142, in luft 5685. 5883, of fless 495. [107]. 11891; vgl. anch muchedel 7719 , bi suench $962 *$. 4810.

Gen. flesses 4504 .

Anm. 1. Von $s e ̨$ (ae. $s \hat{e}$ m. f.) begegnen nsg. se, see 3. 998, asg. (?) in se 10610 (:aze), dsg.(?) in(to) se 916.925 (:aze).

B. Plural. Die normale endung im nom., acc. (voc.) und dat. ist $-s$ bei den kurzsilbigen, -es bei den langsilbigen: hupes huften (n) 6580, metes (a) 3454 , studes, stedes (n) 1659, (a) [83], vor stapes 9416 , in studes (d?) 2751 ö., mid speres $1120.7475-$ duntes (n) 2591 ö., gistes (n) 6002 ö., wormes (n) 1007 ö., among gustes 5788, to wormes 10045.

Anm. 2. Zum sing. $b e n d$ gehörten im Ae. als plurale bendas und benda, bende (Sievers $\$ 261$, anm. 2). Dementsprechend finden wir bei Robert: in bendes (a?) 134i. 7119. 10016, mid bendes 2691 und in bende (a?) $7120 \mathrm{~K}$, of bende $7793 \mathrm{~K}$. (Vgl. of pis bende : sende inf., St. Juliana v. 190).

Anm. 3. Wie Wealas und Norpwegas sind auch Seaxe, Angle, Norphymbre singulare geworden: acc. al Westsex 5204, al Soupsex 5205, Estangle 6035, dat. (?) of Westsex, of Estangle, of Norphumber 4662 ö., of Nurphomber 59 (wegen des $o, u$ dieses letzten namens siehe diss. § $32 \mathrm{a})$.

$\$ 81$. 2. Feminina.

A. Singular. Es sind nur ursprunglich langsilbige wörter vertreten. Non., ace., (voc.) und dat. endigen gewöhnlich auf -e: nede (n) 3471, (a) 1360. 4751. [4] R ö., (d?) in nede $1763 \mathrm{R}$. 7103 R. $73566 \mathrm{R}$ - dede die tat (n) 28J9 R. $4011 \mathrm{R}$ ö., (a) 2823 
R. 8015 R 8., (d?) in dede 9326 R. 9383 R ö. - mizte (n) 583 R.

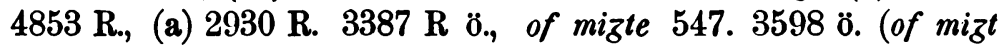
219, wol eine bloss gelegentliche apocope) - sizte (n) 5024 . 7240. 7283, (a) $1624 \mathrm{R}$ (vgl. 792), of sizte 3187 R. 6209.11178 $\mathrm{R}$, bi insizte $6261 \mathrm{R}$ - munde (ae. -mynd, gemynd fem. $\mathrm{u}$. neutr.) (n) 9544 (: of kunde). 10844, (a) 2066, (d?) in munde 3041. - Nur im nom. und dat. trifft man ferde (uerde) (n) 438*. 7920*, (d?) in verde 4406, nur im acc. und dat. tide (a) $7605 \mathrm{R},(\mathrm{d}$ ?) in tyde $1963 \mathrm{R}$. $3961 \mathrm{R}$, atte tide $6562 \mathrm{R}$, heste (ae. hôes) (a) 2319. 6588. $10813 \mathrm{R}$ ö. (biheste acc. 6606. 8719), (d?) to heste 3118 R. $4387 \mathrm{R}$ und zifte (a) $9673 \dagger$, (d?) mid zifte 7113, nur im acc. eizte die habe 11108. - Dazu kommen zwei wörter, welche auf verschiedene ae. bildungen zurtickgehen können: quene (ae. $c w e ̂ n$ st. und cwêne sw.) (n) $6808 \mathrm{R}$. 7010 R ö., (a) 610 R. 10111 R ö., (d) 769 R. - kunde (ae. cynd neutr., gecynde neutr., gecynd fem. neutr., gecyndu sw. fem.) (n) 251. 3524. 6979, (a) 2065. 2104 ö., (d?) of kunde 1080. - Nur mit einer praep. (poru) begegnet bisokne (ae. sôcen) 10174.

Anm. 1. Von sizle sind im nom. und acc. auch endungslose formen vorhanden: sizt (n) 6282, (a) 4191 (:to-nizt), vgl. insizt (a) 817. 3290 (: al nizt).

Anm. 2. Ein nom. ned begegnet in gewissen wendungen, welche bedeuten 'es ist nötig': as ned was 2569, bote it n. were 3054, zif it $n$. were 2494, zif n. were to .. 6908, zwanne gret $n$. is 3053 , as it gret neod were 2283 , po mest ned was $8799^{*}$.

Anm. 3. In den mit tid gebildeten bezeichnungen kirchlicher fest. tage, wo dieses tid nur einen nebenton trägt, ist dasselbe gewöhnlich unflectiert geblieben, auch in (mutmasslichen) dativen wie at Sein Bartelmeus tid 11938, at S. Nicholas tid 10471, at S. Simondes tid 11801, at Seint Hillari tid 11456, fram Seinte Margarete tid 11408, of Witeson(e) tyd 3111*.3117*, at, to alle halve(n) tid 8601. 10162. 11808. Ganz vereinzelt sind aboute, at Seint Andreves tide 6343 R. 6484.

Anm. 4. Das ae. spêd ist nur durch einen endunglosen asg. sped 2710 vertreten, von ae. benc trifft man atte bench 12021 und a-benche (pl.?) $2525 \mathrm{R}$.

Anm. 5. Ueber wizt, $a(u) z t, n a(u) z t, o(u) z t, n o(u) z t$ s. 165.

Ein genitiv ist quene 608. 4503. $7064 \mathrm{R} \ddot{0}$.

B. Plural. 1) Auf -es: nom. acc. nedes 10855 , dedes 6849 . 7436, verdes 4170, bihestes 4750, fusles fäuste 7085, ziftes 1363 . 2460 ö., wiztes elfen 2750, quenes 3935; - dat.(?) of dedes 6552. 11841 ö., of ziftes $1155.2373 \ddot{0}$. - 2) Auf -en: acc. hiden häute 8337 , worten wurzeln 6999 . 
VIII. Ae. $u$-stämme.

1. Masculina.

\$ 82. Kurzsilbige. Vertreten sind ae. sunu, wudu, spitu.

A. Singular. Nom., ace., voc., dat. gehen auf $-e$ aus; nom.: sone 5812 R. $8914 \mathrm{R}$ ö., wode 5842 ; - acc.: sone $5938 \mathrm{R}$. $9618+$; - dat.: sone 9124 (: binome pp.). 4586, to s. $9149 \mathrm{R}$, in wode 1282.1964 , mid spite $4213 \mathrm{R}$; - voc.: sone 5754.6974 ; gen.: sone $9836^{*}$. 9854*, sones $1610.7524^{*} .9845$.

B. Plural. Alle vorkommenden casus haben -es: nom. acc.: sones 536. 1422 ö., wodes 3887. 10262; - dat.: sones 5269 . 6881. 7189, of vodes 487. 7679; - gen.: sones 2825. 2892.

§ 83. Langsilbige. Von diesen sind vertreten feld, und ford in städtenamen. Dazu kommen die mehrsilbigen ae. sumor und winter. Die flexion gleicht der der consonantisch auslautenden 0 -stämme:

A. Singular. Nom.: somer 3717. 10960, winter 3735. 7655 ö.; - acc.: veld 4536. $9015 \mathrm{R}$, Hereford 67, Bedeford 69 (Bedeuorde 10683!), winter 5291. [121]; - dat.(?) of, in velde 5374 R. $7407 \mathrm{R}$ (out of welde $1004 \mathrm{R}$ ) neben of, in veld 1147. 1950 ö., toward, in Chaster-, Chastorfelde 11854.11866 neben of Chastelfeld 11851; to Guldeforde 6704, at, of Herevorde 142. 10203 neben of Oxenford, of Hertford $103 \mathrm{f}$; to somere 10590 neben in somer 8609. 11085 ö., at missomer 10881; in winter 8595, at midewinter 9043. - Gen.: winteres 1288.

B. Plural. winter erscheint unflectiert, wie im Ae.: winter (a) 5267, feld bildet feldes (n) 7799, in feldes (d?) 3963. 3972.

2. Feminina.

$\$$ \$4. Von kurzsilbigen begegnet nur nose (ae. nosu) in dem ausdruck maugre is nose 2090 (: Teolose), (tiber doren, dores pl. s. $\$ 69$, anm. 6); von langsilbigen flor (ae. flôr) im nsg. 5831 und hond, welches folgendermassen flectiert: asg. hond 741:3 R, vgl. 402. 573. 1840. 6746. 10053, dsg.(?) in hond 583. 3724 R. 4366 R. 9965 und vielleicht auch honde, falls of, in, mid honde 348 R. 7077. 6276 R. $7309 \mathrm{R}$ singulare sind. Ueber den (singularischen oder pluralischen?) instrumental is owe honde s. $\S 61$, anm. Sichere pluralformen sind die accusative honde 6105. 9966. 11729 (in der verbindung vet \& honde) und wol auch 4078. $6182 \mathrm{R}$, honden 7079. 7186. 7439. 9342. 9344 und der dativ(?) mid honden $7208^{*}$. 
IX. Ae. $n$-stämme.

$\$$ 85. In den verschiedenen casus des sing., abgesehen vom gen., gehen die ae. $n$-stämme bei Robert auf $-e$ aus. Bei den fem. hat anch der gen. diese endung, die masc. bilden denselben auf $-e s$, von neutren fehlt ein beleg. Die formen des plur. endigen teils auf $-e$, teils auf $-e n$, teils auf $-e s$.

1. Masculina.

A. Singular. Nom:: ssrewe $9531 \mathrm{R}$, frogge 1562 , hare $9384 \mathrm{R}$, fere (ae.gefêra) 283, wille $2491 \mathrm{R}$, name $371 \mathrm{R} .8896 \mathrm{R}$, wone $7828 \mathrm{R}$ - halwe (substantiviertes adj.) 4792; - acc.: hawe 10767 R, biliue 10197 (ae. bîl eofa msc. oder bîlifen fem., s. Bosworth-Toller), stẹde ross 8178 ö., onde (ae. anda) 937, wille $9758 \mathrm{R}$, name $8902 \mathrm{R}$, time $8537 \mathrm{R}$; - dat.(?): into mave $6363 \mathrm{R}$, of biliue $827 \mathrm{R}$, to more wurzel $7255 \mathrm{R}$, in name $1403 \mathrm{R}$, in wone $4718 \mathrm{R}$; - voc.: ssrewe 2721 ; - instr.(?): is owe name $654 \mathrm{R}$. Gen.: ssrewes 1889, popes 9713†. 10163. 10305. 10368. 10419 (der von Sturzen-Becker, 'Some notes on the leading grammat. char. etc.' p. 19 erwähnte gen. pope v. 7409 steht nur in B, A hat popes).

B. Plural. (Nom. acc. dat.) Endung -e: yuere socios 5994 R. - Endung -en: nom. acc.: portreuen 11205*, ssrewen 5281. 5289. 9975 ö. (vpe pe ssrevene! 8415, acc.? - BC haben hie und da ssrewes), louerdsuiken 6399 , oxen 5570 , assen esel 8337 - halwen 5346 ; - dat. (to) slerren $2436^{*}$. 4703 , of halven 1928. 5098. - Endung -es: nom. acc. bowes schiessbogen 11222, dropes 11746 , times 191 , wrecches halunken 4455 ; - dat. of popes 1919 , wrecches 9918 . - Neben steden rosse (a) 3823 steht stedes (a) 7489. 11551, vpe, of stedes 8412. 11562. - Genitive pl. sind alle halwe 8061.9537. 11432 ö., alle halven 8601. 11214 (sc. churche) und vielleicht portereues 11223 (vgl. v. 11204 f.). Versteckt ist auch ein alter genitiv in Oxenford 70 ö. (ae. Oxnaford).

Anm. 1. Ae. lêoma und plega verlieren die endung und flectieren wie o-stämme: nsg. le(o)m 3178. 8603 8.; - asg. pley 5395. 7296; dsg. (?) of, mid pley 5514 (: wey viam). 8053 (: Mounbrey npr.) neben of pleye 3972 (: nobleye); - npl. leomes 3180.

Anm. 2. Dass die form nekk asg. (vor cons.) 11159 auf einem blossen schreibfehler beruht, zeigt die setzung des kk (auslautende gemination wird, wie wir sahen, sonst stets vereinfacht, vgl. z. b. auch nad 7034* statt nadde) und die schreibung nekke 7709*. 8267 (asg.).

Anm. 3. Neben Porlesmoupe (a) 3436. 8761, (d?) at P. $5223 \mathrm{R}$ i. 
steht of Porlesmoup $4764 \mathrm{R}$. Die ae. form des namens ist Portes $m \hat{\imath} p u$, doch lag es nahe, $m \hat{u} p a$ mit $m \hat{u} p$ zu vertauschen, vgl. Temese mous 1097, hauene moup 477.

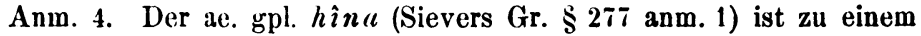
sw. msc. geworden: hine nsg. 9919, asg. $10521 \mathrm{R}$; hinen npl. 11198.

$\$$ 86. 2. Neutra.

A. Singular. Nom. eye auge 6952 R. $8388 \mathrm{R}$; - acc. eye 1609. - B. Plural. Endung -e: acc. (bope is) eye $6716 \mathrm{R}$. 7258 R. 7713, dat.(": pl.?) to ere zu ohren 10118 (: pere da). Endung -en: nom. eyen 5513, acc. eyen 7185 ö., dat.(?) of, mid eyen 4144. 5348 .

\$ 87. 3. Feminina.

A. Singular. Nom.: tlye fliege $8815 \mathrm{R}$, sope seife 143, prute 3409 R. 11702 , hauene 1470 , sonne 330 , tunge 270 ; acc.: mowe (ae. $m \hat{a}$ g/e) $6458 \mathrm{R}$, soupside $10547 \mathrm{R}$, chirche $4787 \mathrm{R}$. $9206 \mathrm{R}$, messe 7237; - dat.(?) in side $3623 \mathrm{R}$, at masse $10433 \mathrm{R}$, of erpe 1323, of nonne 8943, in hurne $7353 \mathrm{R}$; - gen.: (in an) hauene (moup) 477 (B $\alpha \beta \gamma$ : hauenes), Holi-Churche (rizte, ping) $9677 \dagger$. 9772†, heuene (bliss) (= ae. heofenan bliss ggg. oder heofena bliss gpl.?) 5651, (in) Temese (grounde) 1171, (at) Temese (moup[e]) 1097. 5248. - Bei manchen verbindungen kann es zweifelhaft erscheinen, ob der voranstehende genitiv und das denselben regierende substantiv nicht schon fur das sprachgefuhl Robert's zu einem wirklichen compositum verschmolzen waren: churche peis 11118, Lammasse day 8669 ö., Misselmasse day, eue, nizt 6044. 8792. 11650 ö., sonne lizt 8655, sicher war dies der fall in sone(n)day 8724. 9909, Witsoneday 3118, Witeson(e)tyd 3111. 3117, Witsoneneue 11855.

B. Plural. Nom. acc.: Endung -en: ameten 5984, massen 8368. 11321. 11677, herten 9336, bellen 10485, colfren 3937, laddren 3103\%, neddren 1008*. - Endung -es: hauenes 1744, sides 11561. - Dat.(?): Endung -en: mid laddren 8490, out of liperen 8124*. - Endung -es: toward hauenes 11347. - chirche schwankt: chirchen (n) 2801. 4645 ö., to ch. 7868*, chirches (a) 3975 (so oft in B, z. b. 1812).

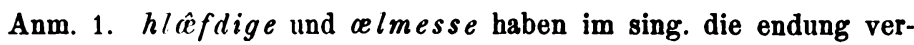
loren: leuedi (n) 3280. 3309, (a) 4365. 9252, (d) of l. 3614. 7808, (g) 6044. - of almes (B: almesse) 6780, vgl. das compositum almesdede 10251. 10815. - Der plur. zu leuedi heisst leuedies, z. b. 3275. 3969.

Anm. 2. Von ae. hosu, spadu begegnen dsg. (?) in hose 2654, to spade $2197 \mathrm{R}$, apl. a peire hosen 8013*. 
$\$ 88.4$. Contractionen.

Die ae. contractionen $f \hat{a}$ 'feind' und $t \hat{a}$ 'zehe', za denen sich das aus $f(\hat{a} n$ entwickelte $f l \hat{a}$ 'pfeil' gesellt hatte, sind durch folgende formen vertreten: A. Sing. Nom. fo $7894 \mathrm{R}$,

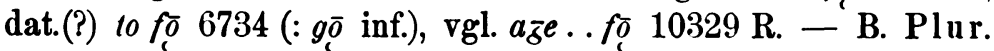
Nom. acc. fōn 5424 R. $10084 \mathrm{R} ; 4855 \mathrm{R} .11672 \mathrm{R}$ und noch sehr oft; flōn $8123 \mathrm{R}$; - dat.(?) fram, of fōn 2704 R. 7916 R. $10698 \mathrm{R}$ u. s. w.; mid flōn 7475 ; to tộn $11177 \mathrm{R}$. Man sieht, der plur. hat ein festes $n$. Nur zweimal begegnet als plur. $f_{\bar{c}}: 3571$ of pe Saxons is fo (: $w 0$ das weh) und 3400 of is fo (:go inf.). An der zweiten stelle könnte man fon:gon schreiben, aber an der ersten lässt sich nichts ändern. Konnte vielleicht, wie in anderen sprachen, der sing. 'feind' collectiv für den plur. eintreten? - Hier sei auch das substantivierte adverb $w \hat{a}$ erwähnt, welches das alte $w \hat{e} a$ verdrängt hat: nsg. $w \bar{o} 1198 \mathrm{R}$, asg. $w \bar{o}$ 3020 R. 4654 R, d(?)sg. in, of, to wo 3437 R. 1725 R. 9909 R.

$\$$ 89. 5. Ae. abstracta auf $-u$.

Nom., acc. und dat. des sing. endigen auf -e. Der gen. ist nicht belegt. Nom. brệde 7229, hệle (ae. hôe $l[u]$ ) [106], hệte 7182; - acc. ouer, in brệde (dat.?) 7246. 7911, hẹ le 664, elde das alter 241, helde lehenstrene 5805; - dat.(?) to hịle 3164. 8066. [115], in, of elde 2013. 7104; 6426 (vor eld 7785*).

X. Vereinzelte consonantische stämme.

$\$$ 90. 1. Masculina.

a) fôt, $t \hat{o} p$. Sing. Nom. fot $10049 ;$ - acc. fot, vot 6119 . 10057 ; - dat.(?) mid, wib vot 6894.7014 .7015 , vp vot 7734 , an vot 3399. 5887, wat an horse wal auote (ae. on fôtum pl.?) 4271, mid hond and mid fote (ae. instr. fôte) $5620 \mathrm{R}$ - Plu r. Nom. acc. vet 6578.6895 ö., (in) tep 8267. 8404; - dat.(?) at, to vet 5891. 10094; 9021 R. 9240 ö., mid tep 8633.

b) $\operatorname{man}(n)$, wîfman, lêofman. Sing. Nom. man $214 \mathrm{R}$. 1828 R ö., womman 869. 2506 ö.; - acc. man 1399 R. 8069 R*, womman 2734. 5658 ö., lefmon 7069; - dat.(?) of man $10939 \mathrm{R}$, vgl. 3981.5803 u. s. W., in, of womman 2755. 4180. (Statt [wom]man wird oft [wom]mon geschrieben, s. diss. $§ 10$,b.) Ein dat. (of) manne, to monne begegnet 1397*. 8248 (: panne dann). 6285. Gen. mannes, monnes 755*. 4065. 5433. 7527 ö. - Plu r. Nom. acc. voc. men 181. 3900 R. 11271, wimmen 981. 983. 2073. $3959 \ddot{\text { ö, }}$ lefmen 10206; - dat.(?) of men 145 R. 848. 947. 1737 o., of wim- 
men 917. 978. 5079 ö. - - Für den gen. pl. von man begegnen vier formen: 1) Dem ae. manna entsprechend, manne 1073*. 2103*. 2865*. 3447. 7764. 10957*, monne 7872*. 8509*. 8553*.

2) Aus manna und dem nom. ace. men gemischt, menne 186*. 2101. 8839. 10995*. - 3) mannes, monnes, eine form, welche, wie die unten $\mathrm{zu}$ erwähnenden gen. pl. frendes 7347 und operes 5153, beweist, dass man schon anfing, das $s$, bez. es, bei pluralen, deren nomiuativ nicht mit $(e) s$ gebildet warde, als specifisches genitivzeichen $\mathrm{zu}$ verwenden. mannes, monnes = manna gpl. $+s$. Uebrigens ist der pluralische charakter dieser form unzweifelhaft nur in v. 9307: of monie bousend monnes depe encheson he is; in v. 11934 of oper mannes ('von fremdem gut'), v. 2206 in oper monnes hond ('in fremder hand') und in v. 2800 mannes blod ('menschenblut') könnte mannes singularisch gemeint sein (in v. 2206 haben $\mathrm{B} \alpha$ manne, monne). Auch in v. $2752 \mathrm{f}$. beweist der gegensatz wimmen fourme noch nicht, dass das mannes in mannes forme plur. ist. - 4) Mit umschreibung: men is 2936 (pe hepenemen is route), B hat dort mennes, also menne (no. 2) mit dem genitiv-s.

Anm. 1. Das vimmen in wimmen fourme 2753 ist wol nicht genitiv, sondern der ganze ausdruck ist als ein compositum anzusehen, dessen erster bestandteil keine flexion zeigt.

Anm. 2. In der hs. A wird, wie in der ne. sussprache, im allgemeinen streng zwischen singularischem womman und pluralischem wimmen geschieden. Nur 2752 steht fälschlich wommen (B $\alpha$ : wymmen, wimmen), und umgekehrt 11053 wimman (C: womman). Eine verwechslung des numerus von seiten des copisten liegt vor in v. 5735, wo A womman schreibt, während $\mathrm{BC} \alpha$ die richtige pluralform haben.

c) mônap. Sing. monje (n) 1408, (a) 1369. 8445 ö., (d ?) in m. 8236. 8469 ö. - Plur. Acc. six monpes 678. 6654. 7262, aber dat.(?) ar tuelf monpe 7342 .

$\$ 91.2$. Feminina.

a) hnutu. Asg. note 8336 .

b) bô $, \hat{a} c, h r \hat{o} c$ beinkleid, burg, dung, niht, Cent, Wiht. A. Singular. Nom. ök 4240. 5546, nizl 3624.5317 ö; - ace. (on) boc 5395 R. 10370, ok 5110, nigt 1578 R. 3289. 6911 ö.; dat. in, of boc 56. 646. $4818 \mathrm{R}$ ö. neben in boce 138* (mit auffälliger schreibung, man sollte boke erwarten), in dunge ( $u=u$ oder $\ddot{u}$ ?) 6336. 6337, of, at, in nizt 2526. 4140. 9767 R. 11650, bi nizte 3501 R. 10665 R ö., fram day to nizte 788 (: mid knizte dsg.), it drou to nizte $3251 \mathrm{R}$ (aber stets to-nizt 'heute nacht' 
5523. 4192) - of Kent 9474 (: iwent nsg.) - to Wigl $7742 \mathrm{R}$. Das ae. burh, burg ergibt boru (n) 1094, (a) 1620. 8552, (d?) of $b$. 1623. Die flexivische umgelautete form byrig ist in den ortsnamen Kanterburi, Salesburi, Ambresburi fest geworden (vgl. Wright's glossar). - Der acc. brech 6579* (wol singularisch verstanden) beruht auf dem ae. apl. brêc. - B. Plural. boc bildet bokes 1813 (a), in bokes (d?) 5106. nizt bleibt unflectiert, nicht nur, wie im Ne., in den verbindungen seue nizt 3303, fourten(e) nizt 7394. 11010 ö., sondern auch mit anderen zahlwörtern: pre, viftene nizt 2972. $8601 \ddot{0}$.

XI. Ae. $r$-stämme.

\$92. fader, brôpor, môdor, dohtor, sweostor. A. Singufar. Acc., voc. und dat. sind gleich dem nom.: fader (n) 608, (a) 227, (d) 4919, (v) 10456 - broper (n) $5672 \mathrm{R}$, (a) $1722 \mathrm{R}$, (d) 9924 - moder (n) 1545, (a) 227, (d) 5885, (v) 6971 doz̆ter (n) 728, (a) 281, (d) 826, (v) 692 - soster (n) 1695, (a) 3704, (d) 9305. - Auch der gen. zeigt selten flexion: faderes 614 (B $\alpha \beta \gamma$ : fader) neben sonstigem fader, z. b. 2837. 9905, moderes 5906 (BC $\alpha$ : moder) neben moder 1543. $1946 \ddot{0}$. - broper flectiert nie, vgl. 2583.9255 u. s. w.; bei soster sone 3525 fuhlte man das soster vielleicht nicht mehr als gen., sondern bloss als flexionslosen compositionsbestandteil. - B. Plural. fader and moder bilden den plur. auf -es, die anderen auf -en: faderes (n) 2563. $2696 \ddot{0}$., of f. $7427-\bmod (e) r e s(n) 993.2563-$ breperen, brepern, brepren (n) 1627. 7972; 895. $2845 ; 1719$, of breperen 6474.7299 - doztren (a) 6462.7620 , (d) 731 - sostren (n) 721, (a) 7560 , to s. 10910 .

\section{Ae. $n d-$ stämme.}

83. Vertreten durch frend (ae. frêond). Sing.: frend (n) 4878, (a) 848. - Plur.: frend (n) 352*. 1765. 7972\%, (a) 2108*, (v) 2191, of f. 10597. 11843. Der gen. pl. heisst, wie erwähnt, frendes 7347.

XIII. Ae. os-, es-stämme.

84. a) êar, hrŷjer. Npl. eres 10045 - d(?)pl. of muperen 1209.

b) lomb, $\hat{e} g$, cild. Singular. Nom. lomb 1321, child 225; - acc. ey 8334, child 1559; - dat. lomb 5680 (lombe? s. \& 74, anm. 1), of child 9097. 9103 neben mid, azen childe $4934 \mathrm{R}$. 
$6979 \mathrm{R}$; - gen. childes 5687. - Plural. lomb (n) 6447 - children (n) 2564. 2719 ö. neben vereinzeltem childern (a) 2099; of children 918 ö.

Anm. Eigentiimlich ist das lombe in v. 7609: ac be hii arise \& abbep ilurnd fram pe weued hor wombe, wolues dede hii nimep vorp pat er dude as lombe. Es läge nahe, nombes: lombes oder womb' (aus reimnot apocopiert) : lom $l$ einzufiihren, aber keine dieser beiden conjecturen wird durch die varianten gestiitzt. Vielleicht haben $\mathrm{C} \alpha \delta$, welche of lombe schreiben, das richtige; lombe wïre dann entweder dat. sg. (ae. lombe) oder dat. pl. (spätae. lo mbum).

\section{Skandinavische lehnwörter.}

\$95. Von skand. lehnwörtern sind bei Robert folgende vertreten: 1) An. st. msc. (sveinn:) suein nsg. 7456 , swaines $n p l$. 1218, apl. 2195. - (grimr, schlecht bezeugt, s. Kölbing, Engl. Stud. II, 516:) yromes apl. 2214. - (vacngr:) wengen apl. 671*. (leggr:) legges apl. 6942. - 2) An. st. fem. (gaum:) gọme asg. 1316 R. 2719 R ö. - (ván:) wōn nsg. 17 R. 1504 R. 10749 R, asg. 442 R. $8291 \mathrm{R}$, of won 1915 R. $6540 \mathrm{R}$, vgl. iwōn asg. $10790 \mathrm{R} .-$ (bón:) bọne asg. 327.2495 R. -- (rót:) rọten ạpl. 8338. - 3) An. st. neutr. (t im:) tọme asg. 'musse' 11656. - (tipindi:) Dieses hat mit suffixvertauschung tiping, tiding ergeben und sich in seiner flexion den verbalsubstantiven auf -ing angeschlossen: nsg. tidinge, in B typing 1770, asg. tipinge 11575 (: bringe inf.), tidiny 6523 (: king nsg.). Zu dieser singularisierten form des alten pluraletantums wird nun auch ein neuer plural tydinges (a) 5343 gebildet. - 4) An. sw. msc. (fél agi:) felawe nsg. $2143 \mathrm{R}$, asg. $9979 \mathrm{R}$, felawes gsg. 4407 - felawes, felaus apl. $2393.2335 \ddot{~ o . ~}$

\section{Wörter zweifelhafter herkunft.}

$\$ 96$. a) Mit consonantischem auslaut: bosk, belegt im plur. vnder boskes 11370 (skand. oder andd.?). -- bost nsg. 4266. 5171 R ö. (kelt.? s. Skeat s. v. boast). - conz, belegt im dsg. of covz $e$ $6395 \mathrm{R}$ (ae. *cohh?). - b) Auf tonloses $e$ endigend: $b i-$ zete gewinn nsg. 1488. (Dieses wort hängt naturlich mit begiet an zusammen, es entspricht dem beieate [nach for], welches sich in den von Bonterwek in Haupt's zeitschrift IX herausgegebenen glossen aus der ersten hälfte des 10. jahrh. [p. 469 ${ }^{b}$ ] findet. Welcher declinationsklasse dieses beieate [= begeate, begeât?] angehört hat, ist nicht recht ersichtlich.) - dręde furcht nsg. 206 R. 970 K. 9389 R, asg. 2945 R. 4338 R ö., dsg.

Anglia. N. F. I. 
in $d .11479 \mathrm{R}$. (Man muss wol als etymon ein ae. stf. *dr $\hat{x} d$ ansetzen, s. Stratmann; in dem adj. dreduol 6137 erscheint das $e$ nach dem $d$ nicht.) - erpgrine erdbeben nsg. 8577. 10934 (C und $\alpha$ haben 8577 erp[e]grene, B schreibt dort erpgrype, $\beta$ erpe dene, $\varepsilon$ erpe den $[$ dene, den $=$ ae.dyne]. Soll man jenes grine, grene von einem ae. $i$-stamm *gryne 'das grunzen, knurren' (vgl. grunian) ableiten? Oder liegt ein zu grânian, ahd. grî$n a n$ gehöriger $i$-stamm *grine zu grunde?) - hẹde obacht asg. $7705 \mathrm{R}$ (vgl. ae. hêdan swv. und hêdd-ern, hydd-ern=*hêdern speicher). - mase nsg. 6585.10236 (ae. oder skand.? s. Skeat s. v. muze). - mōne, imōne klage asg. $801 \mathrm{R} .1195 \mathrm{R}$ (zu $m \hat{\varepsilon}-$ nan gehörig, s. Stratm. s. v. môene). - plodde (C: pludde) asg. (? nach in) 11077 (kelt.? s. Skeat s. v. puddle). - scolle nsg. 375. 4227 (skand.? s. Skeat s. v. scull). - Hier ist auch das suffix - hẹde zu erwähnen, welches das alte - $h \hat{a} d$ fast ganz verdrängt hat; es begegnet bei zahlreichen wörtern, vgl. z. b. gidihede nsg. $6249 \mathrm{~K}$, wrecchede nsg. $7511 \mathrm{R}$, trewehede asg. $7370 \mathrm{R}$, mŭnhede asg. 8716. (Die herkunft dieses suffixes ist noch unerklärt.) - hod haben nur maidenhod $2108^{*}$ und spoushod $606 *$. 1508*. 7701 .

\section{Flexion der romanischen substantiva.}

\$ 97. A. Singular. Die unflectierte form der französischen lehnwörter im Me. entspricht bekanntlich dem casus obliquus des singulars ihrer afz. etyma, d. h. das flexivische $s$ ist nicht mit hertbergenommen worden, und die den accent verschiebenden wörter erscheinen in ihrer längeren gestalt. Wie therall im Me., so machen auch bei Robert mere (z. b. 11205), auncetre (z. b. 3998), sire (z. b. 1391 R) und das bei personennamen häufig vorkommende fiz eine ausnahme (bar nsg. 11282 ist natturlich nur ein schreibfehler, $\mathrm{C}$ hat barun, und auch in A steht barun als nsg. 11791). Bei socours sah man das stammhafte $s$ als flexivisch an und liess es deshalb weg: socour asg. 11600 (: tour turm). Ueber die bei vielen wörtern eintretende apocope eines auslautenden tonlosen $e$ s. $\S 126 \mathrm{der}$ diss. Neu angeftgt ist ein $e$ in nobleie (diss. $\S 127$ ). Im nom., acc. und voc. des sing. erscheint stets die unflectierte form. Im dat. findet man bei einigen consonantisch auslautenden wörtern hie and da ein $e$ angehängt: atte fine 631 R. 6520 R. $10057 \mathrm{R}$, to fine 8547. 10716 (: ni[e]ne neun) neben atte fin 2034. 4765; 
of toure $8478 \mathrm{R}$ neben to tour 9467 ; of store $285 \mathrm{R} .11900 \mathrm{R}$ (acc. stor 8138); wifoute, in striue 6417 R. $11930 \mathrm{R}$ neben in strif $1695 \mathrm{R} .6730 \mathrm{R}$; vpe pointe $2338 \mathrm{R}$, in no p. $2380 \mathrm{R}$, in eche p. $5475 \mathrm{R}$ (acc. point 8868); poru pis traysone (mit anscheinender verkürzung des ou) $2694^{\circ}$ (: Londone); wip treson[e] 11333 (: Norbhamtone) neben of ireso(u)n 3236 R. $11617 \mathrm{R}$; atte chekere $3965 \mathrm{~K}$. (Vielleicht ist auch das $e$ in of vertue 3077 [neben poru ver/u 1911] flexivisch, s. § 127 der diss.) Diese wenigen fälle verschwinden aber gegentiber den zahllosen unflectierten (mutmasslichen) dativen: of solas 11447, of trespas 6984. 7660, in, of cas 2026. 7026 (alle r. a. was, inas prt.), of peys $5399 \mathrm{R}$, of los 11498, in, of castel 9506. 11427, in torment $1819 \mathrm{R}$, to iugement 1236 (: of Kent), u. s.w., vgl. auch den sicheren dativ mareschal $10081 \mathrm{R}$. - Der gen. ist bei den fem. amperesse 9555. 9578 und aunte 12046 (nach analogie der germanischen fem.) und bei dem männlichen verwantschaftsnamen vncle 2061. 4416. 6741 (nach analogie von fader, broper und besonders sone) flexionslos, sonst endigt er auf $-s$ oder -es: prechors 2527, emperours $1057 \ddot{0}$., emperoures $1057 \ddot{0}$., mareschales 10500, angeles 5111, dukes 2482. 9051, dragons 3180. - worre tyme 5389 wurde wol schon als compositum empfunden.

\$ 98. B. Plural. Im nom., ace., voc. und dat. des plur. ist die normale endung $-s$, auch bei den consonantisch anslautenden wörtern. Nom. acc. neueus 4938, Saxons 3437, dragons 2792, paiens 2616, Saracens 5592, Romeins 1442, amirails 8290 , messagers 3526 , leuours 3103 , traytours 6147 - aposile-s 1585*, cable-s 3102, deciple-s 4783, membre-s 11731, prince-s 8289 , rente-s 2462, lettre-s 6864 , anguisse-s 7204 , relike-s 5098 , yle-s 2805, u. s. w. - Voc. sire-s 6822. - Dat. Romeins 1942, Brulons 4676, of barons 2669, of squiers 7753 - prince-s 3855 , of robe-s 3745, of yle-s 3903, in bataile-s 4904, atte table-s 3965.

Anm. 1. Ein auslautendes $d, t$ schwindet meist vor dem $s$ (diss. $\S 150$ b, $\gamma \delta$ ), $f$ dagegen bleibt: bailifs (n) 10267, (d) $9693 \dagger$. NB. Das baylys in B 2730 ist nicht, wie Wright meint, plural, sondern das $s$ steht dort fälschlich für $f$. Die quelle (Gottfried von Monmouth) hat ad praefeclum urbis, und A schreibt dementsprechend richtig baylif. Wenn es dann im nächsten verse heisst pat hii ( $\beta$ : pei) pe moder ... to pe king sende, so darf man das hii nicht auf das wort baylif allein beziehen, sondern es hat mehr den sinn von 'man' und weist auf die ganze stadtbehörde als eine mehrzahl von individuen hin. Uebrigens haben $\mathrm{B} \delta$ he, was freilich in B anch pluralischen sinn haben künnte. 
Anm. 2. Die wörter chatel und menestral haben im plur. eine form mit vocalisation des $l$, welche direct auf den obliquus des afz. plurals zurïckgeht: chateus (diss. $\S 118 \mathrm{c}$ ), menestraus (diss. $\S 161 \mathrm{a}$ ). Sonst ist $l$ im stammauslaut vor dem plural-s erhalten: mid mangenels 11911, mid quarels 1119, cardinals 10376, principals 10204. - Zum sing. castél, der im reime öfters vorkommt (z. b. asg. 9199, d[?]sg. in c. 9506), gehört als plur. castels 2702. 11403, gewöhnlich aber wird castles geschrieben, z. b. 424. 4720. 5121, und dies entspricht dem spätae. cástelas. Jedenfalls bestand auch im sing. die aussprache cástel neben castél. - mesel (1917) bildet seinen plur. nach germanischer weise auf -es, syncopiert aber das $e$ vor dem $l$ nicht, so dass man nicht wissen kann, ob das wort auch germanisch betont wurde: meseles (n) 8960, (a) 8955. - Das afz. ángele ergab im nsg. $\dot{a}(u)$ ngel 5090. 5104. 5113. 5132, im nom. acc. pl. angles (d. h. andžles) 5649. 5653* (B $\alpha$ : angeles). 5684.

Anm. 3. Wörter auf $s$ haben, wie im Afz., kein pluralzeichen: borgeis (n) 11288, Deneys (n) 5442, of D. 5362 R. 5398 R, of Yreis 5551, pe Londreis (d) 9481, vor cors (leichen) 3223, aboute c. 3057.

Anm. 4. Ausser mesel hängen noch folgende wörter im plur. -es statt $-s$ an: a) Einige einsilbige: Gin (der sing. kommt bei Robert nicht vor), Giwes (n) 9913 ö. - duc (nsg. 3430 ö.), dukes (n) 4990. 5013, of d. 5381 - fol (nsg. [adj.?] 6232), foles (n) 3089. 6232 - court (asg. 11341), courtes (a) 1578. 11344 - tour (asg. 8436), toures (a) 8303, of toures 8919 neben of tours 4381 (: honours) - auch von eir erbe (asg. 85i) begegnet einmal eyres (d) $3030^{*}$ neben sonstigem eirs, eyrs (n) 6406, (a) 747. 4913 u. s. w. - b) Die wörter legat (nsg. 10850, vgl. 12000) und prior (asg. sousprior 10144): legates (n) 10276, priores (a) 11962. Diese beiden bezeichnungen kirchlicher würden sind vielleicht direct aus dem Latein entlehnt, jenes sousprior freilich muss seinem praefix nach französisch sein.

Anm. 5. - $n$ haben als pluralendung vncle, chartre, croune und die lateinischen celle, bulle: vnclen (a) 1937, chartren (n) 10253 [chartres (a) 10702], crounen (a) [70], cellen (n) 4808, of bullen 10164.

Anm. 6. partye und aspie bilden ihren plur. teils mit $s$, teils, wie germanische $a$-stämme, ohne flexionszeichen: 431: \& departede hor ost in tuelf parties - 8113: hii departede .. hor ost as in vour partye (r. a. compaynie) - 8244: in six partye (im reim) .. hii delde hor compainye - 9426: in pre partie hii were todeld - 11647 hii adde gode aspies - poru god aspie $11549 \mathrm{R}$.

Anm. 7. Die pluralformen der wörter, welche im sing. auslautendes $e$ apocopiereu, sind in $\S 126$ der diss. zusammengestellt.

Anm. 8. Bei engen verbindungen, wie frere-prechors (nach of) 10105, frere-menors (nach of) 10241, hat nur das zweite glied das pluralzeichen. frere bildet sonst regelrecht freres (nach of, among) 10939. 11316. (Vgl. auch den gen. [?] pl. frere-prechors, -menors 10809. 11388 und den gen.sg. p'erl-mareschales 10500.)

8 99. Der gen. plur. gleicht meistens dem nom., doch findet man bisweilen, dass consonantisch auslautende wörter, 
welche ihren nom. plur. einfach auf $s$ bilden, im gen. -es an den wortstamm fitigen. Belege: lechors 7208 , robbeors 7214, Picars 994, Saxons 2672. 2804, Brutons [123. 129], Romeyns 4427, meseles 8963. - luarones 10510 (npl. barons 3878 ö.), persones 10196 (npl. nicht belegt, nsg. person 9660†), Romaynes 4036, Sarazines 8188 (apl. Sarazins 10902 ö.). Vereinzelt sind bildungen nach germanischen mustern, wie (alre) yle 997, frerene $11320^{*}$.

Anm. Zum npl. Normans it18. 7463. 7469 (vgl. of N. 7500. 7583) gehört als gpl. Normannes 7498. - Man findet übrigens auch poru Normannes 6i00, und mit einfluss des ae. pl. men: of Normens 6701 (vgl. ae. Normenn, Sachsenchron. z. j. 1066).

\section{Personennamen.}

\section{\$100. 1. Masculina.}

A. Singular. Flexion zeigt nur der gen., derselbe geht gewöhnlich auf -es aus, an namen auf unbetontes $e$ tritt nattirlich einfach s: Kimbel-es 1402, Pet(e)r-es 2728.5481.8423, Ion-es 7168, Martin-es 8734, Simond-es 11446, Hillari-es 9562, Andrew-es 8434 - Steuene-s 9145, Arjure-s 3724.

Anm. 1. Die eigennamen anf $s$ haben im gen. keine flexion: Nicolas 757i, Tomas 9818. 9830, Lonis 9599*. Die form Tytusus 1598 beruht wahrscheinlich auf einem dittographischen versehen des copisten, $B \alpha \beta \gamma \delta$ schreiben Tytus, Tilus.

Anm. 2. Als unflectierte genitive muss man nach analogie der anderen von Wright in seinem glossar p. 950 zusammengestellten verbindungen wol auch das Ambrose, Hillari in Saint Ambbrose (C: -es) dai 11334, Seint Hillari tid 11456 ansehen. Dagegen steht der von SturzenBecker l.c. p. 19 erwähnte gen. Harald $\mathbf{2 8 6}$ nur in B, A hat Haraldes.

Anm. 3. Neben Bartclmenes 10466 is. ist 11938 Bartelmens geschrieben.

Anm. 4. Bei der zusammenstellung eines namens mit einem titel wird nach allgemein me. branch nur der eine von beiden bestandteilen flectiert: a) Ohne artikel: Homber kinges 561, king Henryes 9162, king Arpures 3724. (Flexion des zweiten bestandteiles.) - b) Mit artikel, meist mit dazwischentreten des den gen. regierenden substantivs: pe kinges nece Howel 4160, pe kinges sone Ion (: manion) 10612, pe kinges moder. Henri (: spousi) 10650, bi pe kinges day Vortiger 4518 R - bi Gracianes day pe emperor 2013. (Flexion des ersten bestandteiles.)

Anm. 5. In Sein Simondes day (tid) \& sein Iude 10561. 11801 wird der name des ersten heiligen germanisch, der des zweiten lateinisch decliniert, während in Sein Philippes day \& Seint Iacob 8982 der zweite name überhaupt kein flexionszeichen trïgt.

Anm. 6. Ueber umschreibungen des genitivs mit $(h)$ is und franzïsische constructionen s. $\$ 61$. 
Die unflectierte gestalt der französischen eigennamen entspricht nach $\S 97$ dem afz. casus obliquus: Geffray 7094, George 8413 , Guy 9868 , u. s. w. Ausnahmen bilden Hue, z. b. 11720, und mit flexivischem $s$ Giles 11033 , Peris $11755 \mathrm{R} \ddot{0}$. und Iames 8920 (nach of), Gemes 11044. 11136*, of Jame steht 9538, dort hat aber $\mathrm{C}$ das $s$.

B. Plural. be tueie Willames nom. 10908.

2. Feminina.

Bei diesen ist der gen. sg. unflectiert: Emme 7064, Margarele 6468, Eleyne 1991. 4049; - Seinte Marg(a)rete day (tid) 10459. 11408, Seinte Marie eue (tid, churche) 10180. 10952. 11215; mit abfall des e: Seinte Mari day (cue) 7843. 10180.

\section{Adjectiva.}

\section{A. Germanische adjectiva.}

\$ 101. Abgesehen von einigen wenigen gut erhaltenen resten der alten endungen - ne (asg. msc.), -re (dsg. fem.), -ra (gpl.), die sich noch in der flexion von al (s. § 108) und in den erstarrten ausdrticken haluendel 100 ö. (vgl. bi pe haluendel 8025), goderhele 7570, to vroperhele $3420^{*}$. 7282* (neben to wrope [ $\alpha$ : -er] hele 3019) finden, ist als einziges flexionszeichen ein an den wortstamm antretendes tonloses $e$ ubrig geblieben, welches die ae. schwachen endungen und eine anzahl der starken vertritt. Ich gebe zunächst eine thersicht tuber die syntactischen stellungen, in denen die adjectiva vorkommen, mit belegen fur jeden einzelnen fall, um zu zeigen, unter welchen bedingungen das flexivische $e$ angefugt wird. $\mathrm{Zu}$ den belegen verwende ich einsilbige wörter, die auf ae. $o$-stämme zurïckgehen und in ihrer unflectierten gestalt entweder consonantisch auslauten oder auf $e i($ ae. $\hat{x} g, \hat{e} a h)$ endigen. Ueber andere $o$-stiimme, sowie tuber die den tubrigen ae. declinationsklassen angehörigen wörter werde ich weiter unten handeln. Man hat folgende hauptfälle $\mathrm{zu}$ unterscheiden:

\$102. I. Das adjectiv hat unmittelbar vor sich weder den bestimmten artikel noch ein anderes demonstratives oder ein possessives pronomen.

a) Es steht praedicativ.

Singular. Nom.: no ping nas he glad (: adrad pp.) $11272-$ zif per is eny mon so wis $3043 \mathrm{R}$ - he was old $6114 \mathrm{R}$ - pe 
quene was glad 6918 - is moder was dẹd $237 \mathrm{R}$ - so..prout heo was 9479 - it nas nozt god, 10 . $1481 \mathrm{R}$ - nere him no so lop $2692 \mathrm{R}$, u. s. w.

Anm. 1. Verschrieben ist nere he no so proute 1238, wie das reimwort out beweist (vgl. 2329. 6471. 6545).

Acc.: pe king made him vrop inou 378 - (he) made him (somdel) wrop 7790 R. 8024 R - makye him pur blind 7713 (: hind cervam) - (be king ... made it somdel touzt $10498 \mathrm{R})$.

Plural (teils mit, teils ohne $e$ ). Nom.: hii (were) bileued al bare $6258 \mathrm{R}$ - some . bicọme . . blinde oper wode $6064 \mathrm{R}$ bo were per manie wrope $10201 \mathrm{R}$ - pe burgeis were bolde $11208 \mathrm{R}$ - mani (sc. maidens) were glade 2106 - ar we be dẹd $4569 \mathrm{R}$ - tuelf princes .. were dẹd $8289 \mathrm{R}$ - men . . pat .. were god \& wis $9601 \mathrm{R}$ - pe children.. pat were so hey $4896 \mathrm{R}$. Acc.: pis kinges . . mad hom . . wrope $6275 \mathrm{R}$ - he . made is bernes bare $7152 \mathrm{R}$ - pe zales me made vaste 11290 (: agaste pp. pl.) - hii . . robbede pe contreies . \& . . made is sọne bar $11519 \mathrm{R}$.

b) Es steht attributiv.

1. Vor einem substantiv.

Singular. Nom.: zong mon 3477 - an hei man $213-a$ grel sire 1391 - so god knizt 1490 - hei emperesse 9064 a god leuedi 8976 - a gret lond 109 - a smal zerd 510 a foul frogge 1562 - ech gret dede 5820 - so gret martirdom 1817 - gret ssame 8886. - Acc. (mit oder ohne $e$ ): a wīt legat 11920 - an gode sone 1696.1866 - an grete louerding 1771 gret ssame 6097 - gret loue 7102 - strong bataile 3654 - a veir place 416 - a gret $\bar{o} k 510$, cité 1515 , route 1737 - eny foul worm 1006 - grete ssume 1689 - harde hêre 8951 gode chaunce; wille; fourme $9947 ; 11320 ; 11943$ - al longe day 3621. 10491 - an vatte baru (vgl. nsg. msc. vat 8842 , ae. fât $t$ ) 4212 - an yode stude 2705 -- an stronge leuour 2680 - a stronge bataile 3095 ö. - Dat. (vgl. § 60; teils mit, teils ohne $e$ ): a god mon 3981 - a such half-mon 7124 - of a gret duc 3510 10 an heie kinge 6443 - of an so heie kinge 1166 - in gret doute 6697 - in strong martirdom 1878 -- of gret poer 1733 in gret prute; loue 799; 3374 - of god corn; folc 17; 2130 in a vair place 11038 - of rẹde blode 1124 - to grete harme 11267 - of heye kunne 9299 - in gode time 7254 - in wel grete fere 825 - of heye herte 7122 - of grete lore 1603 - 
in gode loue 6326 - fram verre londe 10282 - in vaire manere none 8540 - in an harde stède 2499 - of an so voule pinge 4248. - Gen. (mit oder ohne $e$ ): an hei dukes 9510 - an gode monnes 5998* - an grete erles 10111.

Plural (die vorkommenden einsilbigen wörter haben in allen casus $e$ ). Nom. acc.: zonge sones 1099 - heyemen 8716 (so) gode kniztes 1170.11706 - gode children 2276 - smule ziftes 5394 - wite clopes 9514 - gode cornes; ssipes; lawes $493 ; 1739 ; 3029$ - stronge hous $1170^{\circ}$ - veire weies 169 - folle pritti pousend pound 6009. - Dat. (s. § 60): heyemen 3869 - of opere heyemen inowe 9911 - of greye monekes 9072 - of olde walles 1672 - in gode ssipes 912 - of faire riuers 14 - of fatte weperen 1210. - Gen.: heie menne 2101.

2. Nach einem substantiv. ${ }^{1}$

a) Das substantiv hat den bestimmten artikel oder ein demonstratives oder possessives pronomen vor sich: Belege fehlen.

$\beta$ ) Das substantiv hat keine dieser bestimmungen vor sich:

Singular. Nom.: hardi knizt \& god $11054 \mathrm{R}$ - fers mon \& wod $8630 \mathrm{R}$ - quoyntemon \& bold $1635 \mathrm{R}$ - slazt gret 2142 pité gret 11229.

Anm. 2. Einmal findet man ein $e$ angefligt: 8603 vor per comp fram hire a lem suipe cler \& brizle (r. a. mid rizte). Nach $\mathrm{C} \alpha \beta \varepsilon$ kann im urtext mid rizt gestanden haben. Die hss., welche das $e$ haben, setzten es wol erst unbedachtsam an das rizt und gaben es dann auch dem brizt.

Acc.: an dozter he adde god 6688 - an dozter god inou 6791 - an yle god ( $\$$ riche) ynou 332 - a temple . vair inou 318 - wonder gret 319 - wind gret inou 8487 - wrẹche gret 10038 - fourme god inou 10276 - hii smite bataile strong inou 1199 - b. h. sm. strong inou $1708-$ h. sm. per an b. hard and strong inou 269 ; - mit e: vengance he nom stronge $8836 \mathrm{R}-$ hii . . wolde . . sikernesse vinde gode 2161 R. - Dat. (s. § 60): in batuile strong inou 3628 - to a uair castel \& god $9220 \mathrm{R}$ bi (c. dat.?) tokninge (grisliche \&) gode $11738 \mathrm{R}-$ to be pine of helle stronge $8679 \mathrm{R}$.

Plural (nur einmal ohne $e$ ). Nom. acc.: frend rizt gode $352 \mathrm{R}$ - zeres folle tene 5282 - tounes grete ynowe 2256 walles wide \& stronge inou 417 - \& zef hom . ziftes (riche \&)

1 Adjectiva in solcher stellung künnen bisweilen auch appositiv aufgefasst werden. 
gode $2609 \mathrm{R}$ - hii were . ibrozt in bendes stronge $7119 \mathrm{R}$; ohne $e$ : damaiseles gent \& vair inoz 6250. - Dat.(?): of knizles zonge \& olde $3483 \mathrm{~K}$ - of wellen (swete \&) colde inouz 15 in pines wel stronge $10525 \mathrm{R}$ - of condlen longe \& grete $5889 \mathrm{R}$.

c) Es gehört appositiv zu einem substantiv oder pronomen.

Acc. sg. fem.: an dozter ichabbe of gret pris, noble \& god also 281 (vgl. oben b 2). (Es steht elliptisch mit dem sinne eines temporalen oder concessiven nebensatzes:) Nsg. msc.: he bigan zong ynou to kupe wat he wolde be, pat so zonge is fustes adrou $7084 \mathrm{f}$. $-\mathrm{D}($ (?)sg.: he wolde pat hii adde drede of is bodi ded wanne hii aliue adde $2619 \mathrm{f}$. - Das zonge ist höchst befremdlich, zumal da vorher zong steht. - (Von quic [§ 120] begegnet 4166: he . . al quic [apl.] hom vret.)

d) Es wird substantivisch gebraucht. Apl.: ne... old ne zonge 4934 (von einem mehrsilbigen adjectiv npl. msc. manie Englisse 10999).

$\$$ 103. II. Das adjectiv hat unmittelbar vor sich den bestimmten artikel oder ein anderes demonstratives oder ein possessives pronomen.

a) Es gehört attributiv zu einem substantiv, welches dabei steht oder zu ergänzen ist.

Singular. Nom.: pe gode Brut; knizt 214; 464 - pe wise man 655 -- pe heye emperour 1063 - pulke grete duc 3435 pi gode cosin 2019 - is zonge sone 1869 -- pe gode Cordeile; dozler $736 ; 845$ - pe vaire leuedi 3280 -.. pis gode folc 441 pe sope wei 1264. - Auch eine so enge verbindung wie halfmon 'feigling' (\%. b. 7124) flectiert im inneren: pe haluemon 5793. - Ohne $e$ : pe gret yle; evel; despit $32 ; 185 ; 423$ - be vair biker, the vair asaut 11258. - Acc.: pe stronge king 273 pis (be) gode knizt 1161. 1845 - pe gode Cordeile; Margarete; quene 740; 7566; 6644.7568 - is leue dozter 857 - be grete wo 578 - pe olde heinesse 1065 - bis grete slazl 2056 - pe volle bite 4214 - pis foule best 1564 - is foule caroyne 4421 fi leue bodi 695; - ohne $e$ : his gret ax 573 und (vielleicht dat.) in be bar erpe 6945 (vgl. auch von quic [\$ 120]: is quic eizte 11108). - Dat. (\$ 60): pe olde king 9749 - pe gode manne 8248 to be heye emperour 1060 - is zonge sone 7095 - of pe gode quene 2033 - 10 me (fem.) olde wrecche 4199 - in is foule wille 2534 - in pe faire place 10667 - in is faire halle 9099 - 
in pe grete sę $940 ;-$ ohne $e$ : in is wod rage 4415 (vielleicht ein compositum, wie das deutsche 'tollwut', vgl. das adjectiv wodwrop 6109 und das in $\S 132,2$, anm. 2 uber fol-hardi, fol-large, fol-largesse gesagte). - of be voul $(\mathrm{C} \alpha$ : foule) asaut 7814 . Gen.: be gode Constantines 1992 - bis olde monnes 755 .

Plural. Nom. acc.: pis heie men 2191 - min heie gŏdes 694 - pe proute (sc. men) $8830 \mathrm{R}$ - hor felle messagers 3447 pe hote babes 661 - pe grele walles 422 - alle pe gode (sc. lawes) 9808 R. - (Dat. nur von mehrsilbigen adjectiven zu belegen: Englisse men 5259.) - Adjectiva mit dem artikel hinter eigennamen: Willam pe Rede nsg. $9641 \mathrm{R}$, dsg. 7827 - Seint Eleyne pe gode asg. 1861.

b) Es wird substantivisch gebraucht.

Nsg. msc.: pe wyse $2278 \mathrm{R}$, fem. pe wise $9513 \mathrm{R}$. (Apocope zeigt das lehnwort sley = an. slógr: be sley $7405 \mathrm{R}$.) - Npl.: be Frensse 437, dpl. of pe Frensse 435. - Auch die substantivische neutralform, welche nach ten Brink $\S 235$ bei Chaucer stets unflectiert ist, hat in Robert's chronik mit einer einzigen ausnahme (pat sop asg. 720, allerdings im reim zu dop pl.!) immer das $e$ : pat sope nsg. 6937, asg. 2771. 6261. 6937. 7024. (Vgl. auch pat wowe asg. 5143, von ae. wôh - is, ire owe-n-e nasg. $9669 \uparrow .9697 \dagger .6992^{*}$ - be, vre, hor beste asg. 91.2420. 2972.)

8 104. III. Das adjectiv erscheint im vocativ.

In diesem casus ist dasselbe bei Robert immer flectiert, auch wenn der bestimmte artikel oder ein demonstratives oder possessives pronomen nicht unmittelbar vorhergeht. Sing. leue sone; fader; dozter 5754; 10456; 809 - Sire heie king 2409. (Plur. ze zonge men 3100.)

Anm. Auch in den beiden sützen it (sc. pe suerd) was 'Rede Dep' (quelle: Crocea Mors) icluped 1142 und pat swerd .. pal 'Rede Dep' was icluped 1164 ist Rede Dep, wie es scheint, vocativ, obschon an cine directe anrede des schwertes kaum gedacht wird. - Man hat vielleicht ein recht, viele der sogenannten nominative, welche sich im Ae. bei activer und passiver construction nach clypian, hatan, nemnan finden (s. Koch, Engl. Gr. II $\$ 127,1$ und Mätzner, Altengl. Wb. s. v. $h \hat{a}(a n)$ als vocative aufzufassen, ausser etwa in sätzen wie he clypode God his gefylsta, wo das his anzeigt, dass die benennung nicht mehr eine directe form hat. Jedenfalls war aber der voc. in solchen fallen das ursprüngliche, denn die directe rede und anrede geht in der entwicklung aller sprachen der indirecten rede und benennung voraus. - Das Ae. setzte tibrigens (s. Koch l. c.) bei den verben des nennens in activen constructionen den namen auch in den acc.; in einem satze wie hii clu- 
pede me 'Sam-dęde king' 3415 kann man deshalb nicht entscheiden, ob sam-dede king acc. oder voc. ist. Auf eine directe anrede wird übrigens auch hier, wie es scheint, nicht bezug genommen, vgl. v. 3406 .

$\$ 105$. Im Ae. wurden die adjectiva in den fällen unter I stark, in den fällen unter II schwach flectiert (uber einige ausnahmen von diesem gebrauch, welche aus den denkmälern der älteren poesie zu belegen sind, vgl. Lichtenheld: Das schwache adj. im Ags., in Haupt's zs. XVI, 325-393). Die adjectiva im voc., denen der bestimmte artikel oder ein demonstratives oder possessives pronomen nicht unmittelbar vorherging, hatten teils starke, teils schwache flexion.

Daraus folgt, dass wir bei den adjectiven unter II und 111 das in allen casus des sing. und plur. antretende $e$ direct auf die ae. schwachen endungen zurtickzufthren haben. Das $e$ fehlt, wie wir sahen, nur in ganz vereinzelten fällen (meist vor einem vocal). - Bei den adjectiven unter I muss das $e$ auf die verschiedenen endungen der starken flexionsweise zurttckgehen. Im plur. macht diese ableitung keine schwierigkeiten: das $e$ des num. und acc. entspricht dem $e, a, u$ der männlichen, weiblichen und neutralen flexion (uber die letztere s. Sievers $\S 293$, anm. 3). - Beim dat. ist das $e$ wol der rest des alten -um, doch kann sich dieser casus auch einfach an den nom. und acc. angeglichen haben. Eine solche angleichung hat sicher beim gen. stattgefunden. - Im sing. muss manches fraglich bleiben. Das $e$ des acc. vertritt in einigen fällen (z. b. bei an gode sone) vielleicht das männliche $-n e$, welches (nach consonantischem stammesauslaut) mit einschiebung von -e: ene, dann mit apocope des - $\rho$; $p n$ (vgl. haluendel) und endlich mit schwund des $n$ : $-e$ ergeben haben muisste(?), in anderen fallen lebt darin wol die ae. weibliche endung -e fort (z. b. in: grete ssame = griate sceame). Beim dat. entspricht das $e$ wahrscheinlich dem $-\iota m$ der ae. mänulichen und neutralen flexion. - Genitive auf $c$ lassen sich nur von adjectiven belegen, welche vor genitivischen substantiven auf $-e s$ stehen. Das $s$ des gen. ist in diesen verbindungen verloren gegangen, weil es durch das $s$ des folgenden substantivs uberfltssig gemacht wurde. (Deutlich zeigt dies die flexion des zahlwortes $\bar{o} n$, welches seinen gen. absolut gebraucht auf $-e s$, vor einem substantiv stehend auf $-e$ bildet $[\S 135]$.) -- Bisweilen erscheint das adjectiv ohne jede flexion, wie das oben belegte hei in an hei dukes (und oper, 
dessen gen. absolut operes, vor einem substantiv oper lautet [§111]). Das letztere wäre eigentlich das naturlichere. Ueber die herkunft des $e$ als genitivzeichen kann man verschiedener ansicht sein. Will man nicht annehmen, dass dasselbe direct auf das durch die weglassung des $s$ in den auslaut getretene $e$ der alten endung - es zurtickgeht, so kann man darin einfach das stereotype flexions-e sehen, welches die sprache nach aufgabe der ursprunglichen endung dem gen. gegeben haben misste, um ihn wenigstens nicht ganz unflectiert zu lassen.Vor einem substantiv, dessen gen. auf $e$ endigt (churche, ae. fem.) begegnet holi ohne flexionszeichen 9772 (dieses adjectiv flectiert thberhaupt nie, s. §114), andere derartige genitive könnten sein alle richesse ynou 4477 und moche ssame 3460 (s. § 112), doch fasst man diese formen wol besser als accusative auf.

Die flexion kann, wie wir sahen, im sing. nicht nur beim gen., sondern auch beim acc. und dat. ganz unterbleiben, auch dann, wenn das adjectiv unmittelbar vor dem substantiv steht, zu welchem es gehört; für den plur. konnten wir von einsilbigen wörtern in solcher stellung nur flectierte formen nachweisen, aber sonst zeigt ja auch dieser numerus hie und da flexionslosigkeit. Das fehlen des $e$ ist in den verschiedenen fällen verschieden $\mathrm{zu}$ erklären. In singularischen accusativen wie (wonder) gret lebt die alte unflectierte neutralform fort, gelegentlich mag das $e$ aus lautlichen grtinden durch apocope beseitigt worden sein, in den meisten fällen aber, besonders bei den adjectiven, welche dem substantiv nachgestellt sind oder zu demselben in einem praedicativen verhältniss steben, muss man die unterlassung der flexion auf eine logische ungenauigkeit zurtickfuhren. - Der nom. des sing. hat, ausser an den oben angefuhrten, wahrscheinlich fehlerhaften stellen, keine endung, auch einem ae. weiblichen nom. gl adu entspricht, wie wir sahen, unflectiertes glad.

\$106. Nachdem wir so das eigentliche schema der flexion an den einsilbigen o-stämmen, welche sich noch am consequentesten an dasselbe halten, kennen gelernt haben, kommen wir zu denjenigen $o$-stämmen, welche eine besondere besprechung erfordern, und zu den adjectiven anderer ae.declinationsklassen. Zunächst sei noch darauf hingewiesen, dass auch wörter auf -ou (ae. $\hat{g} g, \hat{o} h$ ) flexionsfähig sind, vgl. zum sg. tou $3636 \mathrm{R} .10605 \mathrm{R}$ den pl. towe $5890 \mathrm{R}$, und zum sg. inou 
911 R. 5633 R ö. den pl. inowe 7474 R. 10416 R. 10523 R. Hierher gehört auch der pl. lowe 7543 (vom an. lágr).

$\$ 10 \%$. Unter den einsilbigen $o$-stämmen nehmen folgende eine gewisse ausnahmestellung ein:

1. rigt und luft. - rizt in der bedeutung 'richtig, ordnungsgemäss' liebt in 'schwacher stellung' (man gestatte mir der kürze halber diesen ausdruck) vor vocal zwar die apocope: te rizl eir (erbe) nsg. 862, dsg. 6680. 9124, he rizl eyres (B $\alpha$ : eir) dpl. 3030, aber wir finden daneben pe rizte crouninge 9907 nsg., of pe rizte kunde 3701, to is rizte more 7231; pe rizle (sc. $m e n$ ) apl. $3218 \mathrm{R}$, pe vnrizte lawes 8726 , vgl. auch to rizle liue 8927 in 'starker stellung'. - Heisst rizt dagegen 'rechts befindlich', so scheint dieses adjectiv, und gleich ihm sein gegensatz luft, mit einem folgenden wort, z. b. hond, side, half, zu einem karmadhârayacompositum zu verschmelzen: $d(?)$ sg. in be rizl-alf 4799. 8204. 9780, in pe rizt-side und in be luflside 520. (8208) - in mi rizt-hond 583, in is rizt-hond 7364; - asg. in is rigl-hond 3617, pe luft-side 8206 - apl. zoure riżthonden 9342. Vor (h)alf und hond allerdings könnte das $e$, ebenso wie vor eir, ans lautlichen grunden unterdrickt sein, aber die verbindungen mit side lehren doch wohl, dass wir hier wirkliche composita ohne flexion des ersten bestandteiles vor uns haben.

\$108. 2. Das unbestimmte zallwort al. Dieses hat, wie im Ae., den bestimmten artikel, ein demonstratives oder possessives pronomen nie unmittelbar vor sich, sondern die stellung ist entweder: al, pron., subst. oder pron., subst., al. Im singular bleibt das wort meist unverändert, vgl. asg. 113. 560. 1513. 3323, dat. (?) sg. 56. 125. 134. 306. 725. 1140. 1389. 1918. 2648. [104]. 6787. 8995, doch begegnen auch fälle von flexion: asg. alle vre zonghede 2195 , dat.(?) sg. in alle manere [5]. 6958, an alie wise 2275. 10741, fram alle tene 2174, mid alle gile 6333, to alle sturnhede 7603 , gen. (abhängig von ynou? oder acc.?): alle richesse ynou 4477. Vor ländernamen ist al stets unflectiert: al France asg. 2084, in al Engelond, Normandie d(?)sg. 1022. 7612. - Als pluralform trifft man gewöhnlich alle, z. b. 1206. 1797. 1813. 1939. 2072. 2080, vereinzelt erscheint al, aber nur in verbindungen, wo das wort vor dem dazugehörigen substantiv steht: al Englisse apl. 6547, al pis men npl. 6974, vgl. 1666. 2566. - In dem ausdruck alne 
winter 1364* begegnet noch das alte accusativische -ne, daneben steht alle winter 5291*. [121*] und alle lence 8593* (alle ist wol aus alne direct durch assimilation des $n$ an das $l$ entstanden, nicht auf dem in $\S 105$ angedeuteten wege). Ausserdem hat sich bei al auch die ae. endung - $r a$ des gen. pl. erhalten: alre (B: aller) manne 2865, alre (B: aler) yle 997, hor alre 2217*. 6709 neben alle bing(e) 1565. 6737, alle halwe $(n)$ 8601. 9537 o., alle soule 7670, alle pe operes aller der anderen 5153. Halb adverbiell dient alre, aller, allor zur einfachen verstiirkung von superlativen, z. b. 1020. 4508*. 5220.

$\S 109$. Den ubergang zu den mehrsilbigen $o$-stämmen bildet das in unflectierter form zweisilbige holu (=ae. holh, nur substantivisch $\mathrm{zu}$ belegen), welches flectiert holve lautet: asg. a fourme of bras, al holu wipinne 5020 - apl. holve stones 2779.

\$110. Mehrsilbige $o$-stämme:

1. Wörter auf -iss (ae. -isc). Diese haben in ihrer flexion nichts bemerkenswertes, sie setzen das flexivische $e$ da, wo es bei den einsilbigen wörtern obligatorisch ist, ebenfalls mit grosser consequenz: sing. toward pe Yrisse se 3183, plur. nom. manie Englisse 10999, we Englissemen 5138, pe Englisse 61, acc. pe Yrisse 3095, dat. pe Englissemen 5259. Neben be Welissemen 5140 steht mit syncope des $i$ Welssemen 9275, Walssemen 9433. (Durchgehend ist die syncope bei Frenss.)

2. Wörter auf -ed. Ueber die schwachen participia auf -ed vgl. § 20 . Sonst endigen auf -ed noch wel-ibōned 'starkknochig', nsg. msc. (p)' 8571, und naked (ae.nacod), welches nur unflectiert vorkommt: naked men apl. 9394; in is naked rug 6985 .

\$111. 3. Wörter auf unbetontes -er: luper, siker und das halb pronominale oper, das im Ae. nur stark flectierte. siker begegnet nur im npl., endungslos, 4104 (p) - luper zeigt zweimal flexion: ssephurdes lupere npl. 7210, of pe lujere men d(?)pl. 848, sonst erscheint es unflectiert: I (attr) asg. 621; dsg. 623. 2359; napl. 5053. 8720. 9176. 9649 - II (attr) nsg. 1464. 1539. 1576. 2605. 1595; asg. 2984. 2921. 2612. 1589. 1593.

1 Im folgenden bezeichnet (p) praedicative, (attr) attributive, (aps) appositive und (abs) absolute, d. h. substantivische verwendung. Eine II oder I giebt auf grund der gruppierung in $\S 103$ und $\S 102$ an, ob ein adj. den bestimmten artikel oder ein demonstratives oder possessives pronomen unmittelbar vor sich hat oder nicht. 
5692; vsg. 802. - oper hat im sing. nie ein $e$ : I (attr) asg. 3192 R. 7017 R. 1697. 1861. 6210. 1560. 275; dsg. 4695 R. 8087 R. 1455. (to:) 1982. (of:) 1244. (in:) 2123; geg. 4065 II nsg. 873. 1809. 8091. 8549. 2736. 155. 2064; asg. 1842". 3832. 5759. 9010; d(?)sg. (of:) 2060. 3647. -- Substantiviertes neutrum: 6581. 11777, eni oper 10294, non oper 6350. 10296. - Im plur. tritt $e$ facultativ an. Belege: I napl. 1531. 1767. 9022. 5164. 9155. 2981. 1059. 3198. 6994; d(?)pl. (in:) 2298. (of:) 5316. 4382 - II napl. 3207. 7469; d(?)pl. (of:) 7846. - Wie schon erwähnt wurde, hat oper in absoluter verwendung einen gsg. operes; derselbe begegnet nur in reciproken constructionen: 3816. 4884. 7968 ö. (Das oper v. 3332 beruht wol auf einem textfehler:) operes kann auch gpl. sein, vgl. 5153.

\$112. 4. Wörter auf unbetontes -el. Flexion zeigt uvel: in vuele time $\mathrm{d}($ ?) $) \mathrm{sg}$. 8809 , of vuele zeres $\mathrm{d}($ ?)pl. 5041 , nicht dagegen idel I (p) npl. ydel 4022. 4028 - II nsg. bat ydel lif 4020. (in pe) middel-erd(e) (d[?]sg.) 9053 ist ein karmadhârayacompositum, vielleicht auch middel-ost: nsg. oure $m$. 9008; $\mathrm{d}($ ?)sg. in pe m. 9272. - Das ae. micel ist selten durch formen mit erhaltenem $l$ vertreten: I asg. mochel anguisse 6127 , npl. mochel men grosse leute 2405 - II asg. pe muchel halle 8023 ; d(?)sg. in is mochele wo 5073 (an dieser stelle scheint die volle form des rhythmus wegen gesetzt zu sein). - An allen den anderen zahllosen stellen, an denen das wort vorkommt, lautet es muche, moche, z. b. I nsg. (attr) 1785. 2086. 11147. (p) 5398. 7326. 8125. 8832. (aps) 5172; asg. (attr) 4654. 5035. 6152; dsg. (attr) 1725. (of:) 11229. (to:) 9909. 10155; gen. (?) moche ssame were hii worpe 3460 - II vor pe muche wō 7821*. - Auch das substantivische neutrum heisst muche, moche: nsg. 4528. 4552, asg. 6368. 7001. - Vgl. auch noch muchedel 1441. 7719 ij. - Das $-l$ des ae. $l \hat{y} t e l$ hat dasselbe schicksal gehabt wie das von micel, man findet es nur ganz sporadisch erhalten: I asg. lutel hẹdde 7705 , lutel wule $3796^{*}$; $\mathrm{d}(?)$ sg. in a lutel stounde 1743. 3210*. 4472*. 4841* - II to pe lutel folc 4568. Sonst fehlt das $l$ immer, vgl. lute wule asg. 6460. 7507. $8787 \ddot{0}$., in $(a$, so) lute stounde 1817. 6537*. 7892*. 11561. 11717 und folgende andere belege: I nsg. (attr) 1469*. 1563*. 5780. 7033. 12018. (p) 1488 R. 5785. 8025. 6252. 6385. 9915 R. 10791; asg. (attr) 5871*. 6756. 11122 o.; - d(?)sg. (attr) (al:) 1510. (of:) 5790. 9575. - Das substantivische neutrum lautet ebenfalls 
lute (es vereinigt in sich ae. $l \hat{y} t e l$ und $l \hat{y} t$ ): nsg. 6998. 8029. $10305 \mathrm{R}$; asg. 5015. 9331; meist erscheint es in halb adverbieller verwendung, z. b. 6783 R. 7542 R. 8324 R. 10175 R.

§ 113. 5. Wörter auf unbetontes $-e(n)$. Ueber die starken participia vgl. § 8. In vain (ae. fag en) blieb das $n$ : nsg. msc. (aps) $588 \mathrm{R}$. $1138 \mathrm{R}$ ö., die nebenform fagen aber wurde zu flexionslosem vawe: nsg. msc. (p) 8339. (aps) $10639 \mathrm{R}$, vgl. noch 3800 und den npl. (p) $4447 \mathrm{R}$. - Ae. agen ergab owe, das alte $n$ kommt nur hie und da bei dem substantivischen neutrum zum vorschein: nsg. asg. is, ire owene sein, ihr eigentum $9669 \uparrow .9697 \dagger .6992 *$ neben is owe $9698 \dagger$ (die Thomaslegende hat dort: in his hond), toward is owe 11029. Bei attributiver verwendung des wortes findet man stets die verkürte form: II asg. his owe fader 243 , is owe wif 610 ; $\mathrm{d}($ ?)gg. mid his owe bowe 374; isg. is owe honde 1382. $1440 \ddot{0}$., body 3812, name 654 ; npl. pin owe men 2468. - Die stoffadjectiva auf -en behalten das $n$ : I d(?)sg. mid an yrene spite 4213 , to Yrenebrugge 8224; apl. yrene ssares 6890 , vgl. auch den beinamen Yrenesyde 6084 ö. neben Yrensyde 6130*. 6136* (Edmond Y.). Syncope des $e$ vor dem $n$ zeigt lînen: asg. a linne ssete 8962 . Ueber cristene und hepene s. § 116.

\$114. 6. Wörter auf unbetontes $-i$ (ae. $-i g)$. Flexion begegnet nur bei gidi, und bei dem unbestimmten zahlwort mani im plur.: I (attr) napl. gydie men 3356, monie heyemen 7718. 8716, monye opere 9022, (abs) monye 9496; 3507. 7448, vgl. 11998 - gpl. monie pousend 9307 - dpl. monie opere 9481 - II nsg. pe gidie wrecche 1564*. 1580. - Bei attributiver verwendung ist mani im plur. anch sehr oft flexionslos, vgl. 247. 1601. 1767. 1390. 1296. 2805. (Verschrieben ist der nsg. manie oper knizl 9826.) - Nur unflectiert erscheinen: nedi I pl. (attr) 6780; blodi II sg. 6194; fury II sg. 6917, pl. 6944. 6995; gulti I pl. (p) 6898; holi (attr) I sg. gen. 2527. 9772, pl. 1650. 4743. 4646. 8411. 7135 - II sg. 4767. 5634. 5708. 10714 ; 185. 1837. 6610. 9874; 4960. 5583. 6793. 8382. 6805. 7093. 8076. 7091. 5873; 1932, pl. 893. 8505; seli vsg. 8808, II sg. 6717. 780. 2528, pl. 6446. 6705 ; sori I pl. (p) 9237; weri I pl. (p) 8253; $w[u] r b i$ I pl. (p) 1417. - Ueber eni s. § 167.

8 115. 7. Composita mit tonlosem zweitem bestandteil. Die mit -les, -vast zusammengesetzten wörter flectieren ge- 
legentlich: vsg. bou faderlese ssreve 2721, npl. gultelese men 6705 [neben apl. (p) faderles 2988], studeuaste npl. (p) 11011 $\mathrm{R}$ (neben be sofuast God 5056). Die compositionen mit -vol fuggen nie ein $e$ an. Belege für deoluol, ioiuol, rigluol, sunuol, willeuol: I pl. (attr) 1737. (p) 2261 - II sg. 3189. 2196; 5045. 5300; 245. 786. 1613. 1998; pl. 3602. 5046.

$\$ 116$. 8. Wörter, welche frtuher consonantischen auslaut hatten, bei Robert aber in dem eigentlich flexionslosen starken nom. sg. (und natillich in den anderen casus) ein $e$ angefugt haben: iliche: per nas ... suerd him iliche $1140 \mathrm{R}$, was non (sc. helm) him iliche $3610 \mathrm{R}$ neben he nas no mon ilich (: of Greneswich) 6055 (vgl. apl. wite clopes .. ilich [C: iliche] pe snowe 9514) - cristene, cristine msc. (p) 1605. 1678. 2536. (attr) 1912. 3157. 4912, vgl. cristine mon 2489. 3157 - hepene (p) 1903 neben hejen (C $\alpha$ : hepene) man [127]. - Im plur. findet man ubrigens neben cristine men 2541. 2560.2570 u. s. w. häufig cristenmen, cristinmen, z. b. 1631. 1811. 10953*. - Der plur. des substantivierten cristine, hepene lautet pe cristine 2938, pe hepene 2571.

\$117. Der kurzsilbige jo-stamm sib flectiert, wie im Ae., gleich den $o$-stämmen mit geminiertem wurzelauslaut: sibbe npl. (p) 9551. (abs) 7086 R. - Das contrahierte frẹ (ae. frêo) ist inflexibel: apl. (attr) 537. (abs) $10317 \mathrm{R}$.

§ 118. Ae. langsilbige jo-stämme und ursprungliche $i$-stämme. Diese endigen schon im starken nom. sg. auf $-e$, sind also einer weiteren flexion nicht fähig. I nsg. mse. (attr) wilde 7689 , (p) blipe $2473 \mathrm{R}$, hende $3799 \mathrm{R}$, milde $1321 \mathrm{R}$. $7688 \mathrm{R}$, stille $5877 \mathrm{R} .11811 \mathrm{R}$, sturne $7352 \mathrm{R}$, trewe [111], wilde $1322 \mathrm{R}$ - fem. (attr) hende $8833 \mathrm{R}$, (p) clene $8948 \mathrm{R}$, kene $629 \mathrm{R}$ - süchl. (p) ymone gemeinsam (ae. *gemâne statt ge-

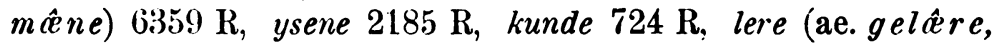
Engl. Stud. VIII, 472) $1800 \mathrm{R}$ (auch statt iler apl. r. a. er 11221 kann man ilere : ere lesen) - pikke $4227 \mathrm{R}$, u. a. m. - riche 1139 ö. vereinigt in sich ae. rîce und afz. riche. - Wörter auf -ede: gretwombede nsg. 7731 , ballede (s. Skeat, E. D. s. v. bald) 7731. 8842 nsg.; dem ae. lâwede entspricht lewede $9677 \dagger$ (vgl. bituene lewedemen $9676 \dagger$ ) und mit apocope lewed $9680 \dagger$ nsg. (apl. lewedmen 9522*).

\$119. Ae. wo-stämme. Das ae. nearu ist durch folgende formen vertreten: I nsg. so nare (B: narw) wey 3312, 
so narw ( $\mathrm{B} \alpha$ : narwe) be brugge was $8286 ; \mathrm{d}($ ?)sg. at a narwe brugge 8285; - pl. among narwe hegges 4299. - Ae. gearu hat inflexibles zare ergeben: I (p) nsg. mse. 6339, sächl. 8516 (: ellesware); asg. msc. 7153 (: bare adj. pl.), sächl. 2484; napl. 4395. 7378 R. 8398. - Das pluraletantum fêawe lebt als vewe fort: nom. (p) $4288 \mathrm{R}$. (abs.) $8674 \mathrm{R}$, acc. frend $v$. $10335 \mathrm{R}$.

\$120. Der $u$-stamm quic (ae. $c$ wic $[u]$ ) flectiert wie die einsilbigen 0 -stämme: vgl. 4166. 7608; 5877. 6428. 11108.

\$121. Das ae. substantiv wicca wurde zum adj.: wikke nsg. (p) $4228 \mathrm{R}$, auch wikked (npl.) begegnet $6899^{*}$. ssreme ist teils substantiv (vgl. § 85), teils adjectiv: 1875. 2091. $6718 \ddot{\text { o. }}$

\section{B. Romanische adjectiva.}

\$122. Die unflectierte form der romanischen adjectiva entspricht dem frz. obliquus sg. msc., nur bei fers ist das flexivische $s$ mit dem wortstamm verschmolzen (wie auch im Agnorm.). In ihrer flexion haben sich die entlehnten wörter den einheimischen angeschlossen. I nsg. (p) hastif 2531, gent (fem.) $566 \mathrm{R}$, pur $184 \mathrm{R}$. $3178 \mathrm{R}$ - asg. (attr) an fole dede 7346, commune rigt 10286, zerd ne fond heo preste non 5888 - $\mathrm{d}(?) \mathrm{sg}$. of an false prệste $9753 \dagger$, in an fole wille 3341 , in vile dep 1584, to so vile dep 6378 neben in pur rizte, meseise 7497. 9245, in cler weder 965, at a certein day, time 1208. 2643 ; - napl. (attr) ferce 5717, (aps) rounde $1172 \mathrm{R}$, (p) false 9380 , preste 383.4838 o. neben (attr) fers 9945 , corleys 2845 $\mathbf{R}$, gentil men (compositum?) 6618, (aps) gent 6258, (p) fers 3910 R. $11210 \mathrm{R}$, gent $1220 \mathrm{R}$, prest 4877 - dpl. $(\$ 60)$ clere sterren 2436, bi certeine messagers 2341 , to diuerse men 7760 , in diuerse studes 8942 , aber wip noble men \& fers $11269 \mathrm{R}$. - Der gpl. begegnet nur von wörtern, die im st. nsg. auf -e ausgehen: simple manne 2103, pouere menne 10995.

\$123. II nsg. pe pure mase 6585 (vgl. pe secunde ost 9428) - asg. is false wif [45], mi fole red (rat) 6233 neben be pur wou 6411, pe commun belle 11215, pat gentil blod 7452 - d(?)sg. (in pe secunde zere 5724) neben of bis vil trespas 10083 - apl. oure puyre fọn 4855 neben is pur fōn 11361.

\$124. Flexionslos scheinen die wörter auf betonten vocal zu sein: I of Welssemen . . pat lizte were \& hardi 9277 - II 8g. is slourdi dede 8528, pl. uor is ardy dedes 2088 - vpl. 
min leue priué kniztes 4363. - Ausserdem können natürlich die adjectiva, deren stamm auf tonloses $e$ ausgeht, wie cointe, quoynle $5474 \mathrm{R} .5508 \mathrm{R}$, debonere $3481 \mathrm{R}$, noble, souple, simple u. s. w., keine flexion zeigen. Abgefallen ist ein solches stammhaftes $e$ bei rebel npl. $1625 \mathrm{R}^{\circ}$ and quit apl. (aps) 11775. (p) 6896.

\$125. Eine femininbildung nach afz. art ist wol das foreine in dem fertig entlehnten ausdruck (into an) chaumbre foreine 633j. (Man vgl. auch 2536 bat he was cristine \& $\mathbf{z}^{\circ}$

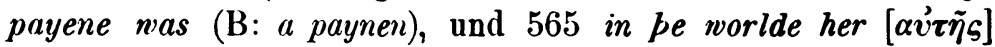
pere nas). - Das adjectiv seint lautet vor franennamen fast immer seinte, z. b. Seinte Marie 3635 ö., Seinte Margarete 11408 ö., in Seint Anne 1927 und Seint Eleyne 1861 hat der folgende vocal den abfall des $-e$ verursacht. Vor männernamen steht 1. Sein: vgl. S. Hue 10103, S. Tomas 9095, S. Richard 10925 - 2. Seint: S. Ednurd 11786, S. Hillari(es) 9562, S. Ion 6150, S. Gregori(es) 6123 - 3. Seinte: S. Peler 1526. 6754, S. Steuen(es) 9145 , S. Poul 1541. - Jedenfalls haben sich hier ae. sanct und afz. saint gemischt. Die form seinte vor männernamen erklärt sich aus dem ae. sancte (= lat. sanct $i$ ), dessen genitivische function man nicht mehr verstand, so dass man dasselbe auch vor heiligennamen setzte, die nicht im genitiv standen. Auch der lat. vocativ sancte mag mit eingewirkt haben. Das seinte vor frauennamen kann auf ae. sancta= lat. sancta oder auf afz. sainte fem. zurtickgehen. Vgl. ten Brink Chauc. $\S 242$ und Pogatscher: Zur lautlehre der griech. u. s. w. lehnwörter im Ae. § 283.

$\$$ 126. Pluralisches $s$ findet man bei adjectiven, welche fertig entlehnten verbiudungen angehören, vgl. canons seculers npl. 5716, harons deserilés 11575, und ausserdem in zwei ausdritcken der kirchenverfassung und des kriegswesens: abbeies pat vacauns were of prelas $9697 \dagger$, hii hulde hom pere defensables 11423.

Anm. Die schreibungen is godes sones npl. 5239 und pe godes knizles npl. 5253, welche Koch (I § 142) auf romanischen einfluss zurlickfithren will, stehen nur in $\mathrm{B}, \mathrm{A}$ hat gode.

\section{Steigerung der adjectiva.}

\$127. Umschreibungen der steigerungsgrade sind bei den adjectiven nur ganz vereinzelt anzutreffen: more milsfol 
9866, mest cruel 8616 und (mit thertragung der flexion auf das mest): of is meste priue men $2343^{*}$.

Sonst lassen sich nur synthetische bildungen belegen. Das suffix des comparativs ist -or $(e)$, -er $(e)$, das des superlativs -ost, -est. Im Ae. endigte der comparativ auf - ra, der mittelvocal bei Robert ist in manchen fällen wol svarabhaktischer natur, ausserdem mag der umstand, dass die adverbia auf -or ausgingen und dass das suffix des superlativs ein $o, e$ enthielt, die veranlassung zur einschiebung des $o, e$ geworden sein. - Beispiele: wisore 3255.8828 , wisost 5388 ö. - wropore 10156 - suiftore 8174 - liztore 1030 - vairor 2506 , vairost 3281 - sikerore 1690 ö. - holyor [18] - weriore 5547 soriore 7051 - rizluolore (l statt $l l !$ vgl. diss. \$ 64) 5391 stalwordore 3960 , stalwordoste 4872 neben stalwardeste 11895 leuere 6288. 7852, leuest 2134 - later 7843 - rapere 5809 lowest (an. lágr) 2438 - kundere 9995* - trinest 859. Umlaut zeigen, wie im Ae.: eldore 750 ö., eldost(e) 684. 4790. 5245 ö., eldest(e) 546. 692 - strengore 354 ö., strengost 351 (zu long sind nur die adverbia lengore 5055. 7967 und lengost 4713 nachzuweisen). - Dagegen findet man (nach mercischer art, vgl. Sievers $\$ 307$ und $\S 310$ ) ohne umlant zongore $1104 *$. 6692, zongost(e) 686. 7622. 7830, zongeste 685*. 710*. - Einige adjectiva verdoppeln bei der steigerung den im wurzelauslant stehenden consonanten; hat die wurzel einen langen vocal, so wird dieser dabei verkurzt: gladdore 7377 , hattore 10961 , braddore 1000*, grettore 4491. 6206, grettere 4794*, grettost 8871, prottore stolzer 9539. - Zu bold gehört baldore 2947. 10487 neben boldore 5238. 9539. 1053́0. - Das g des ae. myrge, welches im positiv vocalisiert worden ist (murie, murye 967. 1579), hat sich vor den steigerungssuffixen erhalten: murgore 3942. 5696, murgost 7162. In ähnlicher weise erscheint bei den adjectiven auf -lich (und bei den adverbien auf -liche) im comparativ und superlativ das ursprïngliche $k$ wieder, ausserdem hat sich bei diesen das alte $\hat{\imath}$ des suffixes, das schon im Ae. zu $\breve{\imath}, \check{e}$ geschwächt worden war (Sievers $\S 43,3$ ), weiter zu $u$ (wol $\ddot{u}$ ) und $o$ gewandelt, wahrscheinlich durch angleichung an das $o$ der folgesilbe (fur welches ubrigens bei Robert $e$ geschrieben wird, sofern der vocal nicht ganz fehlt). So gehört zu dem adjectiv grislich 4141 der comparativ grisloker 11745 (und zu den adverbien liztliche 1761, watliche [v. ae. hwat; 
kommt bei Rob. nicht vor], quointeliche 594: lizlloker 4378, nulloker 7348, quointelucst 2324*). - Der comparativ zu dem selbständigen (i)lich(e) heisst 5680 in A licchere, in BC lyckore. (Vgl. noch Sweet, H. of E. S. § 742.) - Die steigerungsformen von hei sind herre $3625 \ddot{0}$. (= nichtwestsächs. hêrra für westsächs. hîerra) und hecst 10365, hext 127 ö. (= ae. hêuhst, $h \hat{h} h s t)$ neben einmaligem heiest (B: hexte) 1500. - Der ae. superlativ latost (zu leet) wird zu last vereinfacht: pe laste 8081.11342 (: caste prt., vaste adv.).

\$128. god, uvel, muche, lute steigern wie im Ae.: betere 18. 287. 4442 ö. - best 4680 (: west). 8638. 8650 (: forest) worse 10. 846. 1114, wors 7691. 7996. 10176. 10310 - worst 2865 - more 1266. 4505.7021 ö. (: sōre) - mest 1073 (: est osten), sw. meste 10977 (: feste) - lasse 10432 (: Misselmasse). 2827 o. - lest 860. 11974, sw. leste 2102. 3093.

Anm. 1. In der redensart he suor is more op [71]. 8047, die sich auch in der Pilatuslegende (Furnivall, Lives of Saints) v. 187 findet, hat more einen ganz superlativischen sinn.

Anm. 2. Zu more gehört als substantivisches neutrum $m \delta$ : nsg. 9783 (: pio da). 1200 (: wio weh), vgl. wipoute mo $2920 \mathrm{R}$ neben wipoute mor $81 \mathrm{k}$. Dieses mo tritt ausserdem appositiv zu pluralen: mo gistes, 8853 , opere .. mo 2695. 11116, opere munimo $11337 \mathrm{R}$, es hat auch alleinstehend offers den sinn von 'plures' und wird dann mit dem plural des verbs construiert, z. b. 1583 (mo \& mo). 9367. 9397. Vgl. dazu noch he nadde kunnesmen (sones) nammo $7045 \mathrm{~K}$. $78.31 \mathrm{R}$. - Der plural more heisst 'grössere', z. b. none more 151 'keine grösseren', im singular kann more aber auch die menge bezeichnen: nammore asg. 'nicht mehr' 10286 R, vgl. 2820.

\$129. Adjectivische steigerungen von adverbien. $\mathrm{Zu}$ ver gehört verrore 2292 , verrost(e) $3072^{*} .3751$ (ubrigens verwendet Robert ver, wie wir sahen, auch adjectivisch; auch die ae. poesie tat dies, vgl. Sievers $\$ 313$ anm.); nei, das bei Robert nur adverb zu sein scheint (zweifelhafte stellen sind 2285. 4174. 7151. 8656. 9605. 11693), hat einen adjectivischen superlativ next (ae. nêahst, nêhst, nêxt) 128 (: hext); von $\bar{e} r$ ist eror $3424.6642 *$ abgeleitet, von vore verst (s. $§ 147$ ). Superlative auf -mest, -most (= ausserwests. - mâst, s. Sievers $\S 314$ anm. 2): midmeste 685, estmost 4494, sw. westmoste 4495, otemoste (ae. ûtemest) 11433.

$\$ 130$. Die romanischen adjectiva haben, mit ausnahme der oben erwähnten wörter cruel und priué, synthetische steigerung, wie die germanischen: fehlore 3424.7665 ö., chastore 3959 , 
pouerore 7617 ö., purost (B: -est) 2308, noblost(e) 701*. 6346* (B: -est). $\mathrm{Zu}$ dem germanisch-romanischen riche gehört richost (C: -este) 10977.

§131. Flexion der comparative und superlative. Die comparative, welche im Ae. immer schwach flectiert wurden, gehen bei Robert in den verschiedenen casus des sing. und plur. auf $-e$ aus, doch wird dieses $e$ hie und da apocopiert, wie die obigen belege zeigen. Die superlative waren im Ae. starker und schwacher flexion fähig. Sie kommen bei Robert in 'starker stellung' nicht häufig vor, ein einziges mal hat ein solcher superlativ ein flexivisches $e$ : beste red (rat) asg. 3044, sonst sind die superlative in dieser stellung immer flexionslos, auch im plural, vgl. npl. (aps) hext 1660, $\mathrm{d}($ ?)pl. of lest wordes 860 , of feirest men 145 . - Superlative in 'schwacher stellung' apocopieren das $e$ nur selten. Belege: nsg. pe strengost man 351, pe eldost (sc. dozter) 684, pe eldost hous 4790 - asg. pe zongost (sc. dozter) 686, pe uerst ende $7460-\mathrm{d}($ ?)sg. to pe verrost ende 3751 - $\mathrm{npl}$. pe hext 9168 - $\mathrm{d}($ ?)pl. of pe grettost 8871.

Anm. Der zum substantiv erhobene ae. comparativ pl. ieldran, bez. merc. $\hat{a} l d r a n$, eldran, 'vorväter' erscheint bei Robert teils als eldore $907 \ddot{0}$., teils mit erhaltung des $n$ und anfügung eines $e$ als eldrene 249, elderne 2223*. 3708. 9399 und eldorne 4039*.

\section{Adverbia.}

\$132. 1) Bildungen auf - $e$ von adjectiven. foule 11733, harde 1417 (hard inou 1415), heye $10153 \mathrm{R} \ddot{0}$. , ilome $43 \mathrm{R}$ ö., late $6382 \mathrm{R}$, lizte $582 \mathrm{R}$, longe $8852 \mathrm{R}$, rape 2251 , roume 6185, rowe (v. ae. $r u h$ ) 590. 10448 (: howe euch), vaste $256 \mathrm{R}$. $9894 \mathrm{R}$ ö. (vast 1958. 2156), wide $11826 \mathrm{R}$, wrope $729 \mathrm{R}$, lupere 9018 , vuele 6943 (vuel ypaid 2537) - lowe (an. lágr) $6459 \mathrm{R}$. $7312 \mathrm{R}$ - narwe 5954. 10029. - Bei den jo- und $i$-stämmen, deren unflectierte form schon anf $-e$ ausgeht, lautet das adverb gleich dieser: clene $10066 \mathrm{R} \ddot{0}$., stille $2466 \mathrm{R} \ddot{\text { o., }}$ pikke $4649 \mathrm{R}$, ohne umlaut softe $2740 \mathrm{R}$ (für das adjectiv fehlt ein beleg), aber nicht suẹte $8964 \mathrm{R}$.

2) Bildungen auf -liche von adjectiven. folliche gänzlich (neben vol, s. unten) 3694 ö., liztliche leicht 10597, wisliche 432, sikerliche (neben siker, s. unten 3) 2704, quicliche 7471 , baddeliche 11905 , kundeliche 7257 , mit apocope des aus- 
lautenden - $e$ : frelich $6634^{*}$, mit einschiebung von $e$ : hardeliche 2682. 7694, buldeliche 433 (zu bōld), mit ausfall eines $e$ : stalvardliche 8121 neben slalwardeliche 8229 , treuliche 3585 neben treweliche 6108, und mit ausfall von $e$ und gleichzeitiger verkürung des wurzelvocals clanliche 2146. 8942. 9681†. Alle romanischen adjectiva bilden ihr adverb auf -liche: falsliche 7270 , fọlliche töricht 6852 , villiche 2681 - priueliche 595 quointeliche 594, (richeliche 7725) - mit einschiebung von $e$ : feinteliche 10596, scarseliche (v. scars 6862) 10614, aperteliche 7695.

Anm. 1. Ueber hastiliche, hasteliche $\mathrm{s}$. $\$ 141$ der diss., uiber die adverbia der adjectiva auf consonant $+l e$ vgl. $\$ 161$ a der diss.

Anm. 2. Zu pur existiert ein adverb purliche 'ganz und gar' 1512. 3323; zur näheren bestimmung von adjectiven wird jedoch nicht dieses, sondern ein einfaches pur verwendet: pur blind 7713 'ganz blind', $p$. clene 8948 , p. gidy 1542, p. hepene 7540, p. zovit 182, p. versse 8187, p. mesel (adj.?) 1917. Es ist interessant, zu beobachten, dass das afz. $p u r$ in dieser selben weise auch in den keltischen idiomen in der bedeutung 'ganz', 'sehr' vor adjectiven, und ausserdem noch vor participien des passivs, vor adverbien und vor substantiven anzutreffen ist; vgl. Whitley Stokes, Philol. Soc. Transact. 1868-9, p. $21 \overline{f f}$. (106 beispiele aus dem Kornischen) und Owen Pughe, National Dict. of the Welsh lang. s. v. pur. - Nicht adverbiell ist wol das fọl in fol-hardi 9386 (quelle: audaciae irrationabilis) (= ne. fool-hardy), fol-hastif 9393 und fol-large 8001. 8006, sondern wir haben es mit einer engen juxtaposition zweier adjectiva zu tun, wie in dem oben erwähnten wod-wrop 6109, vgl. in Wright's ausgabe appendix $\mathrm{H} 62$. - fol-large findet man auch bei Chaucer (Morris III 338), und dieser bildet dazu ein substantiv fol-largesse (Morris druckt fole-largesse) ebenda (man vgl. die lesarten der einzelnen hss.).

3) Casusformen von adjectiven. Acc. sg. neutr.: al $10905 \mathrm{R}$ ö., vol 2014 ö., inou 7700. $8264 \mathrm{R}$, inis $542 \mathrm{R}$ ö., wis 7024, ney 'beinahe' 2055. 2789 ö. (neiwat 'beinahe' 1791), 'nahe' $167 \mathrm{R}$ (per nei), quic 'schnell' 1647, rizt $2826 \mathrm{R} .6340 \mathrm{R}$, siker 1264. 3838. 4202. 8951. 10357 (immer im sinne von 'zweifelsohne', 'in wahrheit', das oben belegte sikerliche bedeutet 'ohne gefahr'), muche, moche 1384. 2282. 3304 ö., lute 1467*. 5643. 8855 (einmal lucel 11598*). Das adverb zu god ist wel 10089 $\mathrm{R}$ ö. (über welle pat s. $\$ 15 \mathrm{~m}$ der diss.), god erscheint selbst adverbiell nur in dem ausdruck alsogod 'ebenso' 3275. 3861. 4616. 5246. 5942. 8595; 3391 R (neben alsowel 11766. 11980). - Zusammensetzungen mit -ward: aflerward $11349 \mathrm{R}$, azenward $11351 \mathrm{R}$, (a)dorward 7470*. 7474. 7482, hiderward 7471, ouerward $10965 \mathrm{R}$, vpward 7471. - strong 3860, dep 1136. 5622* 
(B: -e), verlich 6065. 10472 und reulich $2690(\mathrm{~B} \alpha \beta$ : -e). 5802 gehören nicht hierher, sie haben ein auslautendes $e$ durch apocope verloren, wie die oben angefuhrten hard, vast $\mathbf{u}$. s. w.

Anm. 3. Ein adverbielles oper findet man in dem ausdruck wan it nolde oper gọn 3373 . Wahrscheinlich ist derselbe nach dem muster von wanne il ne may oper be 5862 gebildet, wo oper praedicatives adj. ist.

Gen. sg. neutr. alles 400. 1562, elles 8157. 9258.

4) Verbindangen von adjectiven mit praepositionen. an onywar 2659 ö., an idel 3070 ö., an hey 'oben' $11248 \mathrm{R}$, 'nach oben' 168. $5746 \mathrm{R}$, 'laut' $11709 \mathrm{R}$, an euene (ae. on efen, das adjectiv lautet im Me. in unflectierter gestalt euene, bei Robert begegnen nur oblique casus) $9567 \mathrm{R}$, abrod $7014 \mathrm{R}$, auorpward 393, alte laste 'zuletzt' 407 ö., atte leste 'wenigstens' 2102, vor gọde 'fürwahr', 'im ernst' (vgl.nfz. pour de bon) 2664 R. [118]. 6361. 7000, of inou 'bei weitem' 592. 4563. 6711. 8018, mid alle 2740, wip alle 663 R. 1535 R. 7012 R.

5) Casusformen von substantiven. Asg.eueridel $1735 \mathrm{R}$, somdel 175, muchedel 395. 10045, pe mestedel 5820, noping 1263, nozt $5435 \mathrm{R}$ ö.; instr. sg. nẹde $1223 \mathrm{R} .1582 \mathrm{R}$, sore $919 \mathrm{R}$ ö.; gsg. dawes 3409; dpl. limemẹle 4203, pecemęle $5624 \mathrm{R}$ (ae. -m $\hat{x}-$ lum), zwile $5 \mathrm{R}$.

6) Verbindungen von substantiven mit praepositionen. among $5652 \mathrm{R}$, amidde $24 \mathrm{R}$ ö., aweye $2778 \mathrm{R}$, aliue $8506 \mathrm{R} \ddot{0}$., bliue 'flugg' $1098 \mathrm{R} \ddot{0}$. (B schreibt meist noch bi liue), adoune $3027 \mathrm{R}$, adoun $5751 \mathrm{R} .11515 \mathrm{R}$, und mit weglassung des $a$ - (= ae. of): perdoune $8759 \mathrm{R} \ddot{\text { o., }}$ vp \& doun $528 \mathrm{R} \ddot{0}$. amis $1258 \mathrm{R}$, a day, amorwe 2708, azere jährlich 2209, to-nizt heute nacht 4192.

7) Bildungen auf $-e=$ ae. $a$. sone $1430 \mathrm{R} \ddot{o}$. (son 4122), zute $5782 \mathrm{R}$ ö. (zut $239 \mathrm{R}$ ö.), zare 5867.

8) Eine bildung auf -inge (ae.-inga). allinge 1103.

Die ortsadverbien ( per, wer u. s. w.) missen hier ubergangen werden. Die zahladverbien stehen in $\S 147$.

\section{Steigerung der adverbia.}

\$133. Als adverb des comparativs dient teils, wie im Ae., die verktirzte form auf -or, -er: suijor 8714, later 236, raper 7122. 9419, teils die volle form auf -ore, -ere: vastore 10298, lengore 5055. 7967 , verrore 9011.10225 , vorpore 4253 , belere 773. 5696*. Besondere einsilbige bildungen sind bet $2335 \mathrm{R}$. 
6386 R ö. (verneint no bet 11955), wors (adv.?) 5521, leng 2398 ö. (B hat immer lenyer), ver (ae. fierr) 7653, ner 2594 R. 6568 $\mathrm{R}$ (= ae. nêar), vgl. 870. 3710, er $9144 \mathrm{R}$ ö. neben ere 2894. 3828 (: pere da). Neben les, z. b. in napeles 7692 R, steht lasse 4. 6643 ö. (les wird auch nach analogie von $m \bar{o}$ im sinne von 'wenigere' substantivisch verwendet, z. b. 625); 'neben $m \bar{o}$, z. b. in (n)euer(e)mo 1472. 5601. 8521. 9845. 10021 (: po da), napemo 5154. 10341 (: po da), wird more adverbiell verwendet: 1560. 2160. 3037. 8297 (: sore adv.), vgl. auch nammore 5110 R. 5197 $\mathrm{R} \ddot{o}$. und nanmore (=nân $m \hat{a r e}$, welches fälschlich für $n \hat{a} m \hat{a} r e$ eingetreten ist) 2208. 3698 (: sore), dazu mit apocope nammor 4234 (: bor eber nsg.).

Anm. Der comparativ ner begegnet auch mit positivischem sinne, aber nur, wenn ein gegensatz zu fer (ze.feorr) vorhanden ist, sonst gibt Robert 'nahe' immer durch nei wieder: ver \& ner (nie ver \& nei!) $3755 \mathrm{R} .4688 \mathrm{R}$ und noch sehr oft; wan me wende he were fer, ofle he was ner (: Mortimer) 11540.

Ueber die comparative zu den adverbien auf -liche vgl. § 127.

Pleonastische umschreibungen sind ofter-mo 5337, innore-mo 5200 , vorpore-mo 5529, verbore-mo (statt vorpore-mo, mit einfluss von ver) 6880 , vorjore-more 2464 *.

$\$ 134$. Die adverbia der superlative sind gleich deren unflectierter form, wie im Ae.: lengost 4713, hecst 10365, next 1618, best 5987 R ö., mest 1565 (: best tier). 686 ö. (almest beinahe 1800). Zu er gehört arst 1658. 4211. - Ueber quointelucst vgl. § 127 .

\section{Zahlwörter.}

\section{A. Cardinalia.}

$\$ 135$. Ein(s). Das ae. $a n$ ist vertreten:

1) Durch formen, welche bedeuten 'unus' und auf ae. starke flexionsformen zurtickgehen: Nom. mse. $\bar{o} n(\mathrm{abs})=$ unus $1825 \mathrm{R}$. $2922(o n=$ aliquis, quidam 4279. 5518. 5864 ö., bei einem eigennamen 9197), pe on broper 7016, pe on (sc. emperour) 1808, pat on (sc. vncle) 1937, pat (is) ō broper 6456. 8694; fem. put on (se. dozler) 7623; sächl. on bissopriche $119^{*}$, be on half 92 . 8270 , pe on (sc. dragon) 2786 , pat on (sc. wonder) 152 , per nas rēd bote ōn $10050 \mathrm{R}$, o day 2920, o cler leom 3178, o bissopriche 105, pe o partie 9680†, pat o bissopriche 99*. - Acc. mse. on $(\mathrm{abs})=$ unum 4277 (: fōn). 8268 (: anōn). 4275. 8647, daneben die alte form (Jen) anne 4601. 8266; sächl. on (sc. chartre) 
10702 , on zer 5162, is on eye 1609 , pe on (sc. suerd) 9451 , put on (sc. ston) 10671, o ping 1113. 2474. 9310, pe o dẹl 3856. Dat.(?) msc. of o mon $8979^{*}$, mid o man 832 , to pe on (abs) 8707, sächl. of one pinge 4008, kunde 1051, blode 3524, zere 10033, time 4895, croune [69]; in one daye 11879, kinedom [69], time 10514, womb 5681, manere 3017; at one mẹle 4204, worde 6812 , réde 8989. (one = anum dsg.) - fram be on ende 171, in pe on side 211*, at on time $6250^{*}$, of on (sc. dede) 6553, fram pen on sẹ 2173 - in o zer 10135.10713, pouzt 10492; at o time 4706. 8867, word 10248; in be o castel 10118; mid is o vot 7014; gen. one mannes 7257, absolut: pe ones 5759. 5764 .

$\$ 136$. 2) Durch eine in allen casus gleichlautende form one mit dem sinne von 'solus', welche auf den ae. schwachen formen ana u. s. w. beruht: nom. pou one $6358 \mathrm{R}$, pe pope one 9528. $11819 \mathrm{R}$, pe erl of L. one 11969, Engelond one 7545; acc. is one $(\mathrm{C} \alpha$ : owe dozter 7137 , ir one 'sie allein' 11806, pi bodi one 695 ö. - Oft tritt al vor one, z. b. 2982. 4215.

\$137. Das ae. $\hat{e} n e$ 'einmal' lebt als ene fort 817. 6951 . 8949 (: clene) (vgl. at ene 1077 R. 2130 R. 8496 R), der alte adverbielle genitiv $\hat{x} n e s$ (s. Sievers, nachtrag zu $\S 237,1$ ) als enes 1623. 4058 ö. - 'Nur', 'allein' heisst one (ae. âne) 6302. Ae. for pôem ânum hat vor pe none-s 5795 ergeben (ten Brink, Chauc. § 247). Vgl. noch aton 2451 R. $11533 \mathrm{R}$, anon, a non 274 R. 7162 R.

$\$$ 138. Zwei. tueie (ae. $t w \hat{e} g e n)$ und $t(u) o($ ae. $t w \hat{a})$ vertreten unterschiedslos alle geschlechter und casus. - tueie, tueye, tweie, tweye (fast stets im reim zu beie 'beide'): nom. msc. 2251. 3377 (gektirzt tuey 2399), ace. msc. 1099. 1422 ö., ace. fem. 6462 , ace. sächl. 2779.11174 ; — dat. (?) msc. (nach to) 6252, fem. 731; of children tueye 6928. - tuō, tvọ: msc. nom.

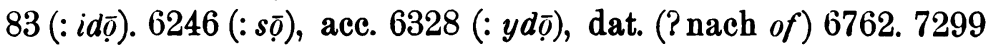
(: sō, alsọ); sächl.nom. 5733, dat.(? nach of) 162, acc. 1626.7184bei jahresnummern 6764. 7105. - Nebenform tọ (: als̄̄) 10739. 11298. - Ueber eine daneben vielleicht bestehende aussprache

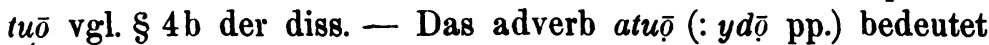
1) 'entzwei' $9787 \dagger$; 2) 'auseinander', ohne den nebensinn des zerbrechens $6153.6323 ; 3$ ) 'in zwei teile' 3854 , vgl. dazu apre 'in drei teile' 9000 und aseuene 'in sieben teile' 4352. 8372.

\$139. Beide. Auch beie (ae. bêgen) und bo (ae. $b \hat{a}$ ) werden unterschiedslos fur alle geschlechter und casus (abge- 
sehen vom gen.) verwendet. Dazu ist bope (an. bábir, nach anderen ae. $b \hat{a} b \hat{a}$ ) gekommen. - beie, beye (meist reimend auf tueie, 4210 auf weye): nom. mse. 1100 . 5288; fem. 6463; acc. msc. 3330. 4210; bō nom. msc. $4702 \mathrm{R} .6742 \mathrm{R}$; bōjee nom. mse. 6274 R. 9503 R, ace. fem. 728 R. 751 R. - Der gen. lautet beire 7980. 11672, bope (hor) 1926.

$\$$ 140. Drei. Die einzige form für alle vorkommenden casus und geschlechter ist pre (ae. prêo nom. acc. fem. neutr.): msc. nom. 4939 R, acc. 536 R, dat. (?nach of) 8682 R; fem. acc. 684; süchl. nom. 9849 , acc. 19. 518 , dat. (? nach of) 6502 R. 6550 R.

$\$$ 141. Die zahlen 4-12 (ausser 8) haben eine flectierte form auf $-e$ und eine unflectierte ohne dieses - $e$. (Bei den wörtern für $7,9,11$ ist das auslautende $-n$ in der unflectierten form geschwunden, vor dem -e der flectierten erhalten.) Wenn eines dieser zahlwörter attributiv vor einem substantiv steht, so hat es meistens die unflectierte form, während es sonst flectiert erscheint. Doch tritt bisweilen die flectierte form fur die unflectierte ein, oder sie wird durch gelegentliche apocope derselben gleich gemacht. Die zahlen lauten: vier: four, vour 3928. 4609 ö., fl. foure 3120.3936 (genitiv) ö. - funf: vif 1169. 1421, fl. viue 38 R. $3137 \mathrm{R}$, fiue $10037 \mathrm{R} \ddot{0}$. - sechs: six 678. 4099 ö., fl. sixe 2810.11943 ö. - sieben: seue, seve $88.7090 \ddot{0}$., fl. seuene 4352. 8372 - acht: eyzte 11009 (: seyte inf., spät-ae. sehtian) - neun: nye 3862. 8086. 10562, fl.nine 9897 R. $10717 \mathrm{R}$. $10942 \mathrm{R}$, nyne $9082 \mathrm{R}$, niene, nyene 8546. 10096 (: fine sb.), mit apocope nyen 5413. 5490 - zehn: ten 1596. 3499 ö., fl. tẹne (: ich wệne) 5282. 102339 ö. - elf: enleue $481^{*} .8782$ ö., endleue 9558. 9570 ö., fl. enleuene 8623. 8859* (B schreibt meistens ellene) - zwïlf: tuelf 431. 11958, fl. tuelue 5325, tuelfe 11965 .

$\$$ 142. Die ubrigen zahlen sind flexionslos. 13-19: prittẹne 1093 (: ich wẹne), prettene $8030 \mathrm{R}$, pretene 10377 - vourtene 5491. 7394 ö. (fourlen nizt 11010. 11518) - viftene, fiftene (: ich wẹ̄ne) 5811. 10488 - sixlene 4907 ö. (: ich wệne). 8074 (: isệne) - seucntene 2044. 6487 (: ich wệne, quệne sb.) - eiztetene 1427. 2504 ö. (: ich wệnu), eigtelen 8981 - nintene 9576. 10646, nientene 1604.

$\$$ 143. 20-90: tuenti 9082 - pritti, pretti $62.10604-$ vourli, fourty 836.4684 - vifly, fifty 4354.5272 - sixti 7174 seuenti 7672 - eizleti 9897 - ni(e)nti 1429.9947. - hond(e)red 6. 453, hundred 1613. - jousend 5963 o., jousund (nur 11879). 
8144. Zusammengesetzte zahlen. Stehen die flexionsfähigen einerzahlen $4,5,6$ vor zehnerzahlen, so haben sie das flexivische $-e$ bisweilen, bisweilen nicht, 7 und 9 kommen in solcher stellung nur flectiert vor. Belege: foure \& tuenty 5414, vour \& tuenti 5591 ö., five \& ffty 9589, vif \& tuenty 1532 ; sixe \& sixli 7260, six \& sixli 7174; seuene \& fourty 4684, vgl. 5272. 6126; nine \& fifty 7842, vgl. 9085. - Hinter der zehnerzahl wird die einerzahl stets flectiert: vifty \& viue 4354, u. s. w. on ist unveränderlich: on \& pritti zer 7519, ninti \& on 9948. Für 2 wird bei zusammensetznngen nur $t(u) \bar{g}$, nie tueie verwendet: tuo \& fourti 1386 , nınti \& tọ 9972 . Neben der decadischen zählweise ist auch die icadische beliebt: four score 478 , six score $(\&$ on) 4800 , eizle score 4526 - four score \& tene 3432, f. s. \& prettene 8030, f. s. \& sixtene 8074, f. s. \& eigletene 8423 (vgl. dagegen ninti \& on 9948, ninti \& to 9972, eizte \& nienti 1429). Auf einer mischung beider zählweisen beruhen bildungen wie sixti \& tene 2687.3636.9736, s. \& endleuene $9799, s . \&$ viflene 5811, s. \& seuentene 2044, welche wol nach afz. vorbilde entstanden sind. (Vgl. daneben seuenti \& pre 7672.)

Bei grösseren addierungen stehen die tausender vor den hundertern, die hunderter vor den zehnern und einern. Die lesart six hondred \& six pousend \& sixti \& sixe 4356 wird durch die varianten berichtigt, es muss heissen six pousend and six hondred u. s. w.

Substantivierung der cardinalia durch den unbestimmten artikel (vgl. Koch I, § 153) ist sehr beliebt und fast fur jede zahl von 2 an zu belegen: an vif zer 9734, wipinne a nye zer 3862 , aboute an tuo zer (wie in ne. dialecten, vgl. Phil. Soc. Trans. 1882-4, p. IX) 7574, an six pousend 4292; ein durch das zahlwort bestimmtes substantiv kann zwischen dieses und den unbestimmten artikel treten: an zeres $t \bar{\emptyset} 9818$ (vgl. an zeres vene 9530).

Einhundert, eintausend heisst immer an hondred, a pousend, nie on hondred, on pousend, auch nicht dann, wenn noch eine kleinere zahl folgt: mid an hondred kniztes 1965, a pousend \& pre 5982, a pousend \& tuelf $6057^{*}$. - pousend kann das substantiv, welches es bestimmt, mit of nach sich haben: pre pousend of wolues 5774, tuenti pousend of men 8235 , vgl. $1209 \mathrm{ff}$. 3536. $4354 \mathrm{f}$. 8235 (neben ten pousend men 10043, six 
p. m. 3497). Nach hondred und den zehnerzahlen findet man nie substantiva mit of.

Subtrahierende zählweise: seue hondred zer bote on zer lasse 5118 (vgl. almest vorte Cristemasse bote tueye dawes lasse 7990).

Brtuche: oper half zer $1 \frac{1}{2}$.jahr 939, a zer \& an half 11164 , prettene zer \& an half 1540, tuo hondred ssipes \& an half 5248.

\section{B. Ordinalia.}

\$145. vers/, sw. verste 57. 192 ö. (adv. verst 44 ö.) secund-e sw. 5724. 9428 ö. - Bei aufzählungen steht oft pe (pat) on-pe oper, auch wenn pe pridde folgt, z. b. 1937. Die anderen (durchgehends schwach flectierenden) zahlen lauten: pridde $23 \mathrm{R} .59 \mathrm{R} \ddot{0}$. - verbe $176 \ddot{0}$. - vifte 1160 und analogisch vifbe 196 - sixte 5018 und sixpe 197 - seuepe $198 * 00$. eizlepe 1860 - nipe 5460. 9720† (Thomaslegende: neozebe) tepe 8934 o., einmal tipe $8935^{*}$ - endleuepe $8440^{*}$ neben enlefte $8578 *$ - tuelfte 7267*. 8606 - 13. - 19. endigen auf -tepe (vgl. diss. \$ 15, l): prittepe 5933 neben prettepe 6069 R. $4782-$ fourtepe $6094 \mathrm{R}$ - viftepe 10708 - sixtepe $6124 \mathrm{R}$ - eiztetepe 6490 - nientepe, nyentepe 5116*. 8699. - 20.-70. (60., 80. und 90. fehlen): tuentipe 5178 - prittipe 9077 - fourtipe $1403^{*}$ - viftipe 5014 - seuentipe 5730*.

Bei zusammengesetzten zahlen wird nur einem der addenden der ordinale charakter verliehen: pe four(e)\& twentipe 540. 5178, pe sixe \&. tuentipe 9063, pe seuepe \& tuenti zer 9068*, in tuelf hundred zer of grace $\&$ pe secunde zere 10121, in pe zer of grace a pousend f fourtepe (sixlepe) 6094. 6124, in pe a bousend zer of grace \& te tepe zere also 6022, vgl. 3112. 6042. 7011.

Ein ordinale zu hondred scheint nicht zu existieren, vielleicht hat hondred sellsst eine ordinale function in jahresangaben wie: in pe endleue hondred zer as in pe zer of grace 8664, in pe endleue hondred zer \& tuenty \& nyne after pat God an erpe com 9082, in pe endleue hondred zer pat oure louerd an erpe com \& pre \& pritti perto. Doch ist es möglich, dass die betreffenden zahlen als blosse nummern einfach cardinal gemeint sind. - Auch zu pousend ist keine ordinale bildung zu belegen, das wort kommt uberhaupt nur in zusammensetzungen nach art der obigen vor, wo ein kleinerer addend ordinal gemacht ist. 
$\S 146$. Von multiplicativen begegnet nur das unbestimmte zahlwort monyvold $(e)$. Ueber die anderen unbestimmten zahlwörter al, mani, lule, muche vgl. oben; feola lebt als uale fort, z. b. 3067 .

\$147. Zahladverbien: ene (s. § 137) - tuie, twie $1301 \mathrm{R}$. 4507 R. 4698 R (verschrieben tueie 6646 r. a. Normandie) - prie $3958 \mathrm{R}$ neben der umschreibung pre sipe 7722 (vgl. das in analogie dazu gebildete ofle sipe 5335).

\section{Pronomina.}

1. Persönliche ungeschlechtige pronomina.

\$14S. ich, $i$ (vgl. diss. \$ 79, anm. 6) - me mir 6973 (: charite). 1347 ö. (: pe dir) - me mich 693 (: pe dich). 9751 (: he er). pou 1888. 7389 (: nou jetzt). Bei invertierten verbalformen wird bou nach dem vorhergehenden auslautenden $t$ der endung zu tou: ssalt tou 4188, meist aber findet völlige verschmelzung statt: artou 2010, hasiow (B: hast pow) 578, misdostou 2721, Jencstow 577, woltou 1889, wostou wolltest du 1339 , und mit schwächung bezw. elision des ou: seiste 10792 , woste wolltest du 3150*, zifst'us 4917 - pe dir 717 ö. (: me mich) - pe dich 692 ö. (: me mich). 3354. 6971.8696 (: priuele, akne, ise sehen). Ueber den reim 10314 be : ze 'ja' vgl. $\S 15 \mathrm{f}$ der diss.; mid pe versehmilzt za mitte 6575 (: sitte). - we 6236 - dat. unbetont us, vs 942. 4995, ous $941^{*}$, betont of vs 2922 , to us 2419 (s. $\S 63,1$ der diss.) - acc. us 1079. 1085. - $z^{e} 4826$ (: be esse) n. s. w., geschrieben $j z e 9360^{1}$ - dat. zou 4381 (of zou 3599), zon 215. 580; ou (C: zou) 10997, on (BC: zou) 4829 - ace. zou 2449. 4368; ou (C: zou) 10997. - hove 'euch' steht 592. 10449. - Die genitive $\min$ u. s. w. werden nur possessiv verwendet, vgl. § 152.

2. Geschlechtiges pronomen der 3. person.

\$149. Singular. Mse. he 1514. 2359. 10480 (: cité, mayné, bise inf.), einmal findet sich im satztiefton $a 3826^{*}$ - dat. (? nach of:) him 214 - acc. him 235 ö., im 2330, hin (B: hym) 1944,

1 Es ist interessant, zu beobachten, dass ein adjectiv, welches praedicativ zu einem singularisch gemeinten höflichkeits- $z e$ gehört, die pluralform haben kann, wenigstens scheint dies der fall $\mathrm{zu}$ sein in dem satze: Sire Emperour, ne be ze no so bolde $1341 \mathrm{R}$, und dort wird sogar die anrede mit pou fortgesetzt. 
vgl. mid hin 2653*; ist ( $J i)$ hum $8629 *$ verschrieben? - Fem. nom. heo 870. 9550 (: leo esse), he 769*. 9248* (vgl. das he in der Sachsenchronik z. j. 1067), zo 635* ö., ze 3349*, sso 615*, sse 709* (B hat meistens heo, he), vgl. diss. $\$ 84 \mathrm{~d}$ - dat. hire 309. 6651 - acc. hire 310, ire 2016, ir 10119. - Neutr. nom. acc. hit 235. 238, it 510. 325 - dat. him 1140. 1141 (bezogen auf ein schwert, das später durch it bezeichnet wird). Plural (ohne geschlechtsunterschiede). Nom. hii 4. 61. 2107. 11907 u. s. w., hi 2407 - dat. hom 50 (und so fast immer), hem 49. 3540 , him $255^{*} .10256^{*}$, Ђam 2167*. 2173* - acc. (gewöhnlich) hom 49 u.s.w., ferner hem 183. 303 (die in B vorherrschende form), him 267*. 2407*. 9405*, zam 1776*. 2174, zem 2751. - Im original lautete wol der dat. und acc. hom, wie A und C meistens schreiben. Dass der copist von B hom vor sich hatte und mit einer gewissen hartnäckigkeit hem, hym dafür einsetzte, zeigen zwei stellen, wo er fur hōm 'nach hause', das er in der eile fur das pronomen hom ansah, hem geschrieben hat: [59]. 5973. (Vgl. ubrigens $\S 84 \mathrm{~d}$ der diss.) - Die genitive des sing. und plur. stehen in $\S 152$.

$\$$ 150. Dazu kommt ein pronomen is, das in accusativischer satzunbetonter verwendung als sing. oder plur. sächliche substantiva vertritt. Belege bei Wright im glossar, vgl. dazu noch 4614. 9667†.11276. Einmal, 282, steht is im sinne von $\alpha \dot{v} \tau \dot{\eta} v$. Vgl. Stratmann, O. E. D. s. v. hes, Sturzen-Becker l. c. p. 42 f. und p. 47 , Wulker, Engl. Stud. II, 125 f.

\section{Reflexivam.}

$\$ 151$. Robert verwendet als reflexiva:

a) Die persönlichen pronomina allein: pe acc. 2723. 5805, zou, zon ace. 428. 2884. 3092 - him asg. 378. 390 ö., dsg. 671. 1691 0.; hire asg. 2605. 6879. 8060, ire asg. 6871. 9470, hire d(?)sg. 9514 - hom apl. 260. 352, hem apl. 321, zam apl. 1776*. 2946*, hom dpl. 343. 416 ö., hem dpl. 875, to him pl. 2394*.

b) Zusammenstellungen mit sulf, und zwar

1) Verbindungen von sulf mit dem acc. bez. dat. des persönlichen pronomens, welche er auch schon fur den nom. eintreten lässt (vgl. Voges, Anglia VI, 328 ff.): (on me sulue 9288 in B), bi vs sulue $1076^{*}$ - nsg. himsulf 745.796 ö., imsulf 1459. 3866, himself 271.407 (das homsulf 7459 ist ein schreibfehler), nit schwacher flexion des sulf: himsulue 6175; fem. hiresulf 
6951 - asg. himsulf 1727, himsulue 4446.4503 ö. - d(?)sg. of himsulue $991^{*}$, to h. 4172.4355, of himsulf 11644, vgl. 7287* apl. homsulue 3358. 9411, homsulf 1487 - $\mathrm{d}($ ?)pl. of homsulue 3491, bi h. 'allein' 2285. 3946 - npl. homsulf 413.

2) Verbindungen von sulf mit dem possessivpronomen. Auch diese werden fur den nom. gebraucht. Nsg. misulf 4009. 8361; bisulf 1082.4918 - asg. misulue 9285 - d(?)sg. on misulue 9288 - (pl. bi oure self 1076 in B).

Die zusammenstellungen von sulf mit dem possessivpronomen findet man auch in nichtreflexiven constructionen, wo nur eine bestimmte person in gegensatz zu einer anderen gebracht wird: vor mi (C: me) sulue 8885, pi sulue asg. 3332. 6301*.

\section{Possessiva.}

\$152. a) 1. und 2. person. Mein, dein: Im sing. steht für alle casus und geschlechter min, pin vor vocal oder $h ; m i, p i$ vor consonanten. Seltener findet sich min, bin vor consonanten, z. b.: asg. $\min$ (sächl.:) 6366*. 6737*. 9772†. 10290; pin (msc.:) 6930*, (sächl.:) 817. 2486. 3995. 4287. 6307 ö. - d(?)sg. (sächl.:) (out of:) $\min 725$; pin (of:) 1891*. 2222*. 3992. (to:) 2484. 2833. (into:) 2011. 2424. (at:) 1337* - gsg. (mse.:) pin 6573. - Nur vor consonanten begegnet pine: $p$. cause asg. 10352 , in $p$. londe dsg. 943 , in b. seruise d(?)sg. $2494^{*}$, of b.reuerye $3991^{*}$, of pine wite brede (brot) 4917. - Im plur. wird vor vocal und $h$ stets min, $\operatorname{bin}$ geschrieben, vor consonanten findet man teils min, bin, teils mine, pine. (Dies gilt für alle casus und geschlechter.) Ganz vereinzelt, und nur vor consonanten, ist $m i$ anzutreffen: npl. 6729*, apl. 7446, vpl. 2191. - Stehen diese pronomina ohne substantiv (praedicativ oder mit ergänzung eines substantivs), so lauten sie im sing. immer min, pin, im plur. immer mine, pine (der plur. mine, pine bedeutet oft 'meine, deine leute') 6571. 705. 723. 287; 699. 335. 1080. 10327. - Unser: oure, vre, bez. ure, und ein wenig seltener vr, bez. ur, werden unterschiedslos vor vocal oder consonant fur alle casus beider numeri und für alle geschlechter (soweit sich belege finden) gebraucht. In praedicativer verwendung trifft man oure 9368. 11712 und auch vre 2963. 4396 (vgl. diss. § 63,1). - Euer: Die gewöhnliche form, attributiv und praedicativ fur jeden casus u. s. w. verwendet, ist zoure. In attributiver stellung begegnen noch zour 2658. 4035, ower 10285. 10310. 11710 und our 5064*. 
b) 3. person. Reste des alten sin sind nicht vorhanden, in allen fällen werden die genitive der personalpronomina zum ausdruck des possessiven verhältnisses gebraucht. - Sing. Msc. his (attr) 374. 1556. (p) 6771 R. $8114 \mathrm{R}$, is (attr) 372. 375; (h)is heisst nicht selten 'seine leute', z. b. $9667 \dagger$, oder 'seine habe', z. b. 11109. - Dieses (h)is wird noch durchaus als genitiv gefithlt, denn es hat nie, wie bei Orm, ein flexivisches $e$. Einmal begegnet eine umsehreibung: in honour of him 466 . Fem. hire (attr) 630. (alleinstehend, d. h. mit ergänzung eines substantivs:) 3944 - ire (attr) 630 ö., hir (attr) 6922, ir (attr) 10223. 11803, here (attr) 732 i., her (attr) 565*. - Plur. hore 'ibre leute' $4984 \mathrm{R}$, 'ihre habe' $10422 \mathrm{R}$, (attr) 251.1152 ö., hor (attr) 265. 266. 2107 i. (hore und hor wiegen in A vor), here (attr) 3551 , her (attr) 249. 274 (here und her werden von B bevorzugt), hare (attr) 621*, jare (attr) 1101*. 8174* (vgl. diss. \& $84 \mathrm{~d}$ ).

Anm. Fin $s$ ist an alleinstehendes oure, zoure, hire, hore bei Robert nirgends angehängt.

\section{Demonstrativa.}

\$153. a) Der bestimmte artikel. Die gewöhnliche form des bestimmten artikels für alle casus u. s. w. ist ein stereotypes $p e$ (vor vocal selten zu $b$ verkurzt: perl 6790 , perl-mareschales 10500 , pemperour 1314). Daneben existieren noch einige alterttimliche formen:

a) Ein dativisches pen (ae. $p \hat{e} m$ ) vor vocal, z. b. 1074*. 2173*. 2882*. 2954*. 3302. 11526 (vgl. auch das oben erwähnte vor be nones). at pen verschmilzt zu atten, z. b. 2363 , wie auch at be atte ergibt: 2838 ö.

B) Ein accusativisches pen, pene, pan, pon, pun (ae. pane, pane, pone) 6348. 9128; 299*; 10057; 2184*; 2690*. Dieses ben u. s. w. steht vor bezeichnungen männlicher personen und auch vor sachnamen, gleichviel ob dieselben mit vocal oder consonant anlauten. ben herrscht in A vor, B schreibt meistens pe, kennt aber pen und setzt dasselbe auch einmal aus versehen für den nominativ (4532), $\mathrm{C}$ hat gewöhnlich pane. Ueber die fälle, in denen diese alte accusativische form anzutreffen ist, gibt nähere auskunft Körner's oben in $\S 62$ erwähnte dissertation p. 35 ff. Körner handelt p. 37 auch uber das dativische pen.

r) Ein neutrales pat, pet, put (nom. oder acc. sg.): pat lif Anglia. N. F. I. 
asg. 9132 , pet maide das mädchen nsg. 4208 , pet worp den wert 7679 , but somer nsg. $10960^{*}$ u. s. w.

Anm. 1. pat, pet, put haben oft demonstrativen charakter und bedeuten 'jener', sie finden sich in diesem sinne auch vor bezeichnungen männlicher personen (ssrewe 4239, traytour 5624) und künnen dativisch gebraucht werden: pal folc 8719, fram put folc 11560*, of pat (put) cas 1844. 6773. Sehr häufig trifft man pat (nie jedoch pet, put) mit der bedeutung 'derjenige' vor substantiven, zu denen ein relatives pat oder ein erklärender infinitiv mit to gehört.

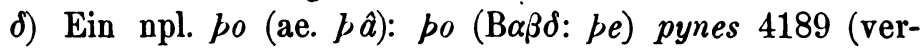
schrieben?).

Anm. 2. $p o$ (r. a. fo feind) begegnet auch absolut vor folgendem pat im sinne von 'diejenigen' 9158. 10328. An 2 anderen stellen findet man den uniformierten artikel $p e$ in gedehnter form in dieser weise demonstrativ verwendet: $p \bar{e}$ (r. a. fri $\ddot{c}$ frei) $p a l=$ eos qui 10178. 10316.

Ausschliesslich demonstrativen charakter haben die alten instrumentale $p e$ (ae. $p \hat{e})=$ 'desto' vor comparativen und $p a n$ (ae. pan): in for al pan 1418, nozt for pan 592. 3315 'trotzdem'.

$\$ 154$. b) pis, pes. Die form pis kommt nur mit substantiven verbunden vor, sie gilt aber fur alle casus beider numeri und für alle geschlechter. Belege: sing. msc. 99. 1161. 7060. 755, fem. 865. 9252. 5968, sächl. 1292. 1192. 571. 1996; - plur. 316. 2105. 296. 429. 7138. 2678. 2116. 2785. 8747. - Das substantivische neutrum lautet immer pis: nsg. 719 R. 840 R. 8906 R, asg. $138 \mathrm{R}$. 7234. $11993 \mathrm{R}$; after pis $9734 \mathrm{R}$, ar pis $6300 \mathrm{R}$. Als gen. sg. fem. ist zufällig nur pes zu belegen (9468). Dieses pes findet sich ausserdem singularisch noch als nom.: pes king 9335 , pes tydinge $4295^{*}$, als acc. pes veiage 4112 und nach praepositionen: in pes cas $8539^{*}$, poru pes signe 1902*, im plural ist es sehr hänfig, es wird in diesem numerus auch substantivisch verwendet: 4129. 6406. 2509*. 9424. 5776*. 1824. 9368. 9048*. 5148 (es heisst stets pes wordes, z. b. 3582.4362 .5720 u. s. w.). - Eine weitere form ist das dativische pisse, pise (ae. pissum sg.), welches vor sachwörtern begegnet: 56. 218. 1694. 1748. 1833. 2743. 3005. 3357. 3733. 3735. 4063. - Von pluralformen sind noch pese (sc. yles) 34 nom., puse (abs) msc. 11757 nom. und pus (sixe) 11950 nom. zu erwähnen.

$\$ 155$. c) pulke 'jener'. Dieses pronomen scheint aus se (be) ilca (ylca) 'idem' und pyllic 'talis' vermischt zu sein; es hat für alle casus die éine form pulke, anch fur den nom. sg.: pulke (sc. man) 1455, b. duc 3455, p. Roberd 9864, p. Bruteine 2095, $p$. (sc. erchebissopriche) 1673 und ebenso (wenigstens 
in verbindung mit substantiven) fur den gen. sg.: p. kinges 3768 , p. childes 5687. - pulke pat heisst 'derjenige, welcher': nsg. msc. 8688, apl. 4303. 9527. - jilke ist 179 geschrieben. - Das alte yl ca lebt daneben noch als ulke fort: pat ulke time 7198, in pis ulke figte $3599 *$.

$\$$ 156. d) sulf 'ipse' erscheint hauptsächlich in den oben (\$151 b) besprochenen reflexiven und nichtreflexiven verbindungen, in denen es teils stark, teils schwach flectiert. Sonst begegnet es noch mit vorausgehendem pis oder bulke, stets mit flexivischem $e$, in der bedeutung 'idem': of pis sulue lond 5399, in pis sulfe zere 5318, bulke sulue seruage asg. 4115, b. s. day, zer asg. 7269.9630 , u. s. w. - self ist 271 , silue 556* geschrieben.

\section{Relativa.}

\$157. a) Das gebräuchlichste relativum ist bat, welches für alle casus des sing. und plur. ausser dem gen. gilt und alle geschlechter vertritt. (Belege für den dativ: sg. 1158, pl. 812.) Besonders häufig steht pat am satzanfang abhängig von einer später folgenden praeposition: pe noblei bat he adde in ibe $800^{\mathrm{b}}$, bulke ost bat pe emperor inne was 1127 , pe kinges sone bat me specp of wide 8738 , u. s. w.

b) Die obliquen casus des sing. und plur. können auch durch formen des ae. $h w \bar{a}$ vertreten werden, gleichviel, ob sich das relativ auf personen oder auf sachen bezieht. Sing. dat. wam msc. 4501, ace. wan 10335 msc., wam 6916 msc.; mit praepositionen: of wam (sc. blod) 1315, of zwam msc. 239, of wan mse. 8560 , of zwan msc. 220 , fram wan (sc. lond) 7561 , poru wan 1631 msc., poru wane 1367 msc., u. s. w. - Plur.: of wan mse. 2696, of wan (sc. lawes) 10700 , mid wan msc. 3356, poru wam insc. 1330. (z)wam geht auf den ae. dat. $h w \hat{e} m, h w \hat{a} m$ zurtick, der im satztiefton gekurzt worden ist. (亏) wan kann sich ebenfalls aus $h w \hat{x} m, h w \hat{a} m$ entwickelt haben, es wäre aber auch möglich, dass es dem spätae. acc. $h w \propto$ ne entspräche, welcher sicher wol in dem erwähnten wane fortlebt. Die beiden casus sind völlig vermengt worden. - Als gen. des sing. und plur. dient was (ae. $h w a s$ ): sg. msc. 4746. 9822; fem. 4195; (sc.ost) 1700 ; pl. msc. 4387. 9771. - Der nom. sg. wo (ae. $h w \bar{a})$ begegnet (mit oder ohne so) nur in der bedeutung 'si quis' 2235*. 4795 und (mit so oder pat) im sinne von 'quicunque' 2445 *. $3603 ; 8338$. In dem satze he esste of is conseilers . . hou he 
mizte best do of pis kinedom, vor he nadde bote an dozter wo mizte is eir be $1975 \mathrm{ff}$. ist wo wol nicht $=\ddot{\eta}$, sondern $=\tau i s$ (abhängig von esste: 'wer sein erbe sein sollte'). 'Quemcunque' findet man $4935^{*}$ durch wanne wiedergegeben, 'cuicunque' mse. durch wan so 10301.10302 (wanne ist vielleicht an anne, nanne angelehnt). - Das neutr. wat (ae. $h w a t$ ) wird bei relativem anschluss verwendet: vor wat, vor $\bar{\delta}$ wat $=$ 'dafur' 1494. 2533; sonst heisst wat 'quodcunque': 2477 (asg.).

c) Ein drittes relativpronomen ist wuch, woch (ae. $h w i l c$ ); es begegnet $\alpha$ ) substantivisch: wuch (woch) so nsg. mse. = 'quicunque' 3967 (vielleicht pl.). 8816, woch apl. mse. $=$ 'qnoscunque' (B: wuche) 2645. 2650. - $\beta$ ) adjectivisch: nur éinmal vor wuch (B: such) dede 1608 .

d) Eine eigentuimliche umschreibung des gen. plur. findet sich in v. 4050: pat of hor beire (von derer beider) blod ich com ( bat .. hor $=$ ae. $p \bar{e}$. heora). pat dient hier als einfache nota relationis. Vgl. Zupitza, Engl. Stud. VIII, 396 und anmerkung zu Guy of Warwick 181-2.

e) An zwei stellen scheint as (vermischt aus me. $a s=$ ae. ealsw $\hat{a}$ und an. es) als relativum in sinne von 'quem' und 'quos' verwendet zu werden: 11183 und 1531. Sonst heisst dieses as nur 'wo' oder 'wohin' (z. b. 1780. 5769. 11324 u. s. w.).

f) 'Utercunque' heisst mejer (ae. $h w a p e r$ ): nsg. msc. 8770, asg. (sc. suerd) 5797, und weper so nsg. msc. 3815.

g) Relative adverbien sind ausser dem erwähnten as noch ber 'wo' 1296. 1470. 4283 ö., pere 'wohin' 2617, und hou so 'wie auch immer' 3363.

\section{Interrogativa.}

\$ 158. A. Substantivisch:

wo 'wer' 985. 2290. 2407. 2735 u. s. w. (B hat meist ho). wan 'Wen' (BC: w[h]am) 6417. to Zwan (B: whom) 709. - wat 'was' nsg. 1185, asg. 576 ö., zwvat 272*. - Handelt es sich darum, individuen aus einer mehrheit herauszuheben, so wird woch gebraucht: me nuste woch was ōn ne woch was oper (quelle: iam nesciebant, quis paganus esset, 'quis christianus) \& hii nuste to wuch truste 2561. - hii nuste woche were hor frend (pl.) ne woche were hor fön 1765 (B: wich, wych).

§ 159. B. Adjectivisch:

a) zwich, wich, wuch, woch (ae. hwilc): sing. nom. woch 
(attr) $3254^{*} .4187^{*}$, wuch (p) 9321. 10457. 11768* - acc. (attr) zwich (sc. contrei) 497, woch 4507, wuch 11813 , wuche (vor stounde) 1360 - dat.(?) (attr) (in:) wuch 7237*. 7331*, woche 5141. (10:) wuche 303. 5628. (mid:) woche 5139; - plur. nom. wuche kniztes were gode (vielleicht ist wuche substantivisch, und kniztes gode gehört zusammen) 3970; wiche (B: wuch) Brutons were 1189.

b) wat (ae. $h w(c t)$ vertritt unflectiert nom. acc. dat. sg. pl., immer attributiv: nsg. wat man 5616, wat red rat 3308, w. ping 2426, vgl. 1403. 2426. 6247 (zwat ist 315. 779* ö. geschrieben) asg. wat ping $2408-\mathrm{d}(?) \mathrm{sg}$. in wat dęp (tod) 2824 , vor wat encheson 2408 - pl. azen wat men 9321*. 9381.

$\$ 160$. 'Uter' heisst weper (ae. hwaeper): A. substantivisch: 8444. - B. adjectivisch: asg. in $w$. ende $3572, \mathrm{~d}($ (?)sg. to $w$. dozter 773 , to $w$. chirche 3942 .

\$ 161. Frageadverbien: wi 2757, wu 4917.7511 'warum', wuwat 6249 'wozu', wanne 7226 , wan 11881 'wann', were 'wo', u. s. w.

\section{Indefinita.}

$\$$ 162. a) Der unbestimmte artikel. Derselbe lautet an vor vocal und $h, a$ oder an vor consonanten. Dieses $a(n)$ ist im allgemeinen flexionslos (belege fur den genitiv: 1331. 2728. 3670. 12013), vereinzelt sind die accusative anne (ae.cenne) und une (ae. Ane fem.): anne stroc 4576, ane wounde $373^{*}$, ane bataile 5312. - Steht der unbestimmte artikel hinter dem substantiv, zu welchem er gehört, so hat er die satzhochtonige form $\bar{o} n$ : zong duc he was on 7096, Roberd pat hosebonde was on (: echon) 11302, hard cas was pat on 5535, acc.: such man on $3152 \mathrm{R}$. Nanchmal trifft man den artikel zugleich vor und hinter dem substantiv: a nonder maister he was on 405, a uair cas it was on 8290.

$\$ 163$. b) $n \bar{o} n($ ae. $n \hat{a} n)$. A befolgt im allgemeinen die regel, dass non im sing. sein $n$ behält, wenn es nicht unmittelbar vor einem substantiv oder vor einem adjectiv, zu dem ein substantiv zu ergänzen ist, steht. Belege: nom. msc. prince non 1973, non niemand 6365, vgl. 2897. 4058; bei sachwörtern: non (sc. wolle) $18 \mathrm{R}, d \bar{e} b$. . non $4391 \mathrm{R}, \mathrm{vgl}$. 3063. 5846. 9377. 9718. 11736; ace. mse. man .. non $316 \mathrm{R}$, non niemanden 4457, vgl. 6435. 10052. $11314 \mathrm{R}$, bei sachw.: stroc non $2683 \mathrm{R}$, vgl. 4042. 6144. 8534. 10669 R. 11763 ; dat. non of hom 10909 ; none begegnet als acc. $977 \mathrm{R}$ : defaute none, $4216 \mathrm{R}$ : targinge none; 
$8823 \mathrm{R}$ : destorbance none, und als dat.(?): in vaire manere none $8540 \mathrm{R}$. Tritt das pronomen unmittelbar vor ein substantiv oder vor ein adjectiv, zu welchem ein substantiv zu ergänzen ist, so hat es folgende formen:

$\alpha)$ Vor vocal oder $h$ : non. Belege: nom. msc. 1723. 4561, sächl. 1415. 4382. 5301. 9310; - acc. msc. 2013. 6786. 7969, sächl. 2671. 3305. 6601. 8461. 8900. 10307; - dat.(?) msc. (to:) 2239, fem.(?) (to:) 3285, sächl. (in:) 2895. 3090. - Vor $h$ ist im dat. auch none anzutreffen: in none hurne 7353 , of none hattore (sc. somer) 10961.

B) Vor consonanten: im nom. meist no: mse. 1641. 2148. 9470, sächl. 679. 838. 1300. 5539, hie und da non 2444. 5109 (vgl. A und $\mathrm{C}$ in v. 8161. 9936).

Anm. 1. Immer steht in A non vor einem durch so bestimmten adjectiv: per nas non so noble prince pat ne dude also 1639, per nas non so wyttimon pat it al vnderstode 3914 , per nas non so heuy charge pat me ne mizte .. bope lede \& bringe $8596 \mathrm{f}$. (acc. me ne vond non so god knizt 5938). Die verse 1639 und 3914 könnte man übersetzen: 'da war niemand ein so edler fürst, ein so kluger mann, dass er'... (vgl. 7716 per nas so heymon non). Jedenfalls sind derartige sätze zuerst in dieser weise gedacht worden, das non wurde substantivisch aufgefasst und vertrat die stelle des subjectes, während das durch so bestimmte adjectiv mit dem dazugehörigen substantiv das praedicat bildete. Auch die accusativische construction me ne vond non so god knizt könnte den sinn haben: 'man erfand niemanden als einen so guten ritter'. Schwieriger ist es, in der verbindung per nas non so heuy charge das non abzusondern, man mulsste etwa construieren: non (sc. charge) nas so heuy charge. Robert hat wol in allen diesen fallen das non bereits an das folgende adjectiv und substantiv angelehnt, so dass diese letzteren seiner denkweise nach nicht mehr praedicat sind, sondern mit dem non zusammen das subject (in v. 5938 das object) bilden; aber die consequente erhaltung des $n$ zeugt dafir, dass man früher das non in der angegebenen weise isoliert hat.

Im acc. findet man no: msc. 10307, sächl. 780. 782. 1170. 2294 ö.; non: non menging 2098*; nanne: nanne fader 2723*, nanne red rat 9121* und vor sachnamen auch oft none: 2769 . 6917. 8715 ö.; - dat.(?) no: msc. (of:) 1972. 3957. 11273, sächl. 413. 711. 2895. 3262 und none sächl. (to:) 3677. 4055. 6779. (of:) 3742. (in:) 6132; - gen. msc. none monnes (pl.?) 6421, sächl. no maner(e) 7392. 8004. 8331. 8342.

Im plural hat das pronomen in allen fällen nur die eine unveränderliche form none (belege: nom. acc. 7306. 2120. 4170. $7544 ; 2212 *$. 151). 
Anm. 2. Schwierigkeiten verursachen die verbindungen des pronomens $n \hat{a} \boldsymbol{n}$ mit $m \hat{a}$ und $m \hat{a} r e$, da zwischen diesen und den verbindungen der negation $n \hat{a}$ mit $m \hat{a}(r e)$ nicht selten verwechslungen eintreten. (Wegen der schwächeren betonung ist das $\hat{a}$ von $n \hat{a} n$ und $n \hat{a}$ verkürzt worden.) $n a$ liegt sicher vor in namore adv. 638*. 2182*. 2819* und na mo 'nicht mebr' (pl.) $527^{*}, n a \hat{n}$ kann den ersten bestandteil bilden in nanmo 'keins mehr' 652. 866, nammo 'keins mehr' 4608 (vgl. namo 641*), nammo 'keinen mehr' 8383, nammo 'keine mehr' (pl.) 8932 (aus nâne mâ?), doch lassen sich diese ausdrücke auch mit 'nicht mehr' wiedergeben, und dann wäre das $n m$ unberechtigt, und ebenso das $m m$, falls dasselbe aus $n m$ entstanden und nicht vielmehr ähnlich wie die italienischen geminationen anlautender consonanten nach vocalisch auslautenden proclitischen wörtchen zu erklïren ist (auf englischem gebiet allerdings wäre dies dann ein unicum). Offenbar falsch ist das $\mathrm{nm}$ in dem adverb nanmore $2208^{*}$. 3683. 3698, für das $\mathrm{mm}$ in nammore adv. 7823. 9522, nammor adv. 4234. 5431. 5432 gilt das eben gesagte.

\$ 164. c) som (ae. sum): sing. acc. som (attr) sächl. 942. 2483. 2802. 3056 - dat.(?) (attr) sächl. som (of:) 3051. (in:) 3804, some (in:) 2703. 3446. 4661; - plur. some (abs) 2106 R. 2214 R. $9426 \mathrm{R}$. (attr) 9302 ; 3975. (aps) hom some 2844 , some pe messagers 2718 , pe tren some 3544 (neben some of is 1293 , s. of is conseilers 1978).

§165. d) Ae. wiht, âwiht, (ôwiht), nâwiht, (nôwiht). wiht lebt fort als wizt 'etwas' $10996 \mathrm{R}$ asg. - dwiht ergab $a_{z} t$ 2225. Der gsg. aztes (nach der ae. neutralen flexion) bedeutet 'von wert': zif hii aztes bep 2247. In constructionen wie: al pe bachelerie pat azt was in pe lond 3798 und al pat azt was 2225 . 7739, wo azt eigentlich substantivisch ist und 'etwas', d. h. 'etwas wertvolles' bedeutet, muss man dieses wort als ein adjectiv mit dem sinne 'wertvoll', 'tuchtig' angesehen haben. Wenigstens ist dazu ein plural(?) azle gebildet worden: stones pat azte (B: aztes) be 3091, auzte men 11999, und in v. 9420 findet sich ein solches azte einem adjectiv beigeordnet: as gode men \& azte (r. a. to nazte; $\mathrm{C}$ hat dort das azte als 'debebant' aufgefasst und deshalb das and weggelassen). Neben auzte men hegegnet in derselben bedeutung 456 wizte men. - ôwiht erscheint als ozt, ouzt, out (vgl. diss. § 48): acc. $9690 \uparrow$. 10464, dat.(?) of ozt 6809; adverbiell: 1384. 6896. 9996 'irgend wie'. $n \hat{a} w i h t$ hat năğt ergeben: nsg. (: slaz̧t sb.) 4302 'nichts', uor nazt (: ycazt) 4371 'mit leichter muthe', to nazte (: azte prt.) 4316 'zu nichte'. - Dem alten nôwiht entspricht nozt, nouzt, nout: nom. 471. 7662 R, ace. 8002 ï., adverbiell 'nicht' 47. 514. 992. 
1385. 9648. 11193; - uor nozt 1) 'mit leichter mthe', 2) 'vergebens': 4378. 9226. 10465. - to nozle 'zu nichte' 2880. 5594.

8 166. e) Ae. $h w \bar{a}, h w c t$. mo, zwo bedeutet 'man', z. b. 758; 24*. - wat - wat $=$ 'teils - teils' 1152.

§ 167. f) Ae. ânig. Die stereotype form des sing. ist eni, eny. Dieselbe kommt teils mit substantiven verbunden, teils absolut im sinne von 'jemand' 3316. 6492. 7555. 7556 oder 'irgend etwas' 6935 vor. Als plur. begegnet éinmal enie: 8835 enie of is men (nom.) neben (attributivem) eni: eni ssipes 1173, eny luper lawes 6613. - Vgl. $\S 15$ a der diss.

\$168. g) Ae. $\hat{\alpha} g(h w a) p e r$ erscheint als eiper: (abs) nsg. 873. 6148 ö. (attr) in eiper side, half (d[?]sg.) 385. $1200 \ddot{0}$. Das reciproke 'einander' wird durch (hor) eiper... ọper (ae. ôper) ausgedruckt: 1720.1756 .3834 u. s. w.

$\$ 169$. h) Ae. $(n) \hat{o} h w a p e r,(n) \hat{o} w p e r$. Auf ôwper geht das conjunctionale oper - oper 'entweder - oder' 9313 zurïck, auch oper 'oder' erscheint oft allein (B verkurzt es schon zu or). - nôw per lebt fort als nọper (r. a. brọpher): hor noper nsg. 4884, asg. $5639 \mathrm{R}$, (attr) nsg. 2834*. 6221, dat.[?] (in:) 4561.

8 170. i) Ae. $\hat{e} l c$ hat ech ergeben: nsg. msc. und sächl. (attr) ech 4660.7033; 525. 1747. 3608, einmal mit -e: eche man 160 (wol ein schreibfehler), (abs) msc. 2654. 4664; - asg. eche man 1399, ech (B: -e) stape 6942; 'jährlich' heisst stets eche zer 909. 3553. 5177 u. 8. w.; - dsg. ech man 1144. 10471, ech prince 1329*; sächl. (attr) ech (of:) 7024. 10406. (in:) 7739; eche (ir:) 1391. 6766. (at:) 2596, u. s.w.; - msc. (abs) ech 2079; - gsg. ech monnes 7230, ech maner 4524. 5561 .

8 171. k) Ae. $\hat{e} l c+\hat{a} n$. Die zusammensetzung von ech mit on ist gewichtiger als das blosse ech. Man findet echon a) singularisch absolut mit ergänzung eines substantivs, z. b. asg. msc. 1016. 1.1640 , vgl. auch 11613 , b) singularisch appositiv bei pluralen: msc. nom. $1764 \mathrm{R}$. $1804 \mathrm{R}$, acc. $1794 \mathrm{R}$. $11360 \mathrm{R}$. Auch absolut of echon 'von allen' 11888 und mit ergänzung eines pluralischen substantivs (stệdes pferde) 11557, vgl. 2688. 10748. - Dazu kommt eine pluralform echone: a) absolut mit ergänzung eines substantivs: acc. msc. 4743.11818 (: one, alone), b) appositiv bei pluralen: nom. nısc. 1196.2981 .4900 , fem. 2119, acc. msc. 694 .

$\$$ 172. l) Ae. $\hat{a} f r e+\hat{x} l c$ (bez. $\hat{y} l c)$ : euerich: (attr) asg. $e$. ende 8330 , e. stape 6950 , dsg.(?) in e. ssire $7677^{*}$, in e. toun 9520 , 
(aps) be princes .. sende euerich to oper 8314, (abs) euerich after oper 8552. - Die form eueri erscheint in eueridel acc. 813, adv. 1735. - Auch euerich wird mit on zusammengesetzt: msc. nsg. $2520 \mathrm{R}$, asg. $2404 \mathrm{R}$.

$\$ 173$. m) Ae. manig $+a \hat{a} n:$ maniōn, moniōn, monyō̄n 'mancher', 'viele'. Dieses pronomen kommt nur singularisch vor, kann aber, alleinstehend, den plural des verbs regieren (z. b. 1824) und appositiv zu pluralischen substantiven treten: mse. nom. 587. 1015. 4845, acc. 317. 613. 10166, dat.(?) (of:) 8710; vgl. 11071. - Vgl. auch noch 2687. 7777 (asg. msc. abs).

$\$$ 174. n) Ae. swilc, swylc erscheint gewöhnlich als such, seltener (besonders in dem letzten teil der lis. A) als suich, swich. Im sing. bleibt das wort meistens unflectiert (vereinzelt ist der acc. suche dede $9241^{*}$ und die dat. [?] in suche nede 3523 , to suiche feste 9910); im plural dagegen fehlt das flexivische $e$ selten: nom. suche (attr) 3067.4750, swiche (attr) 154; acc. (attr) suche 439. 7794, suiche 4285. 11005. 11807; - such nom. (attr) 3052. 3089*. (p) 2417. (C kennt auch eine form swuch, z. b. 11807.)

$\$ 175$. 0) me 'man' (aus dem plur. men verktirzt). Dieses me regiert den sing., z. b. 187. 207. 4684 R. 5157. 10940 R, uber das me sep $183 \mathrm{R} \mathrm{vgl.} \S 21$. Die volle form men 'die menschen', 'man' scheint immer pluralisch aufgefasst zu werden, vgl. 1003. 2782. 5023 (und men ysep 3126 R. 3668 R. 6737 R). Bisweilen hat auch der sing. man eine allgemeine bedeutung, z. b. 159 (B: a mon). 4158. 6410 .

MÚNSTER I. W.

Felix Pabst.

\section{DAS 'TREN'TALLE SANC'TI GREGORII' IN DER EIDINURGHER HANDSCHRIF'T.}

Alb. Kaufuann hat uns im vorigen jahre die mittelenglische legende von der wunderbaren wirkung der Trentale aus fünf handschriften mitgeteilt; seine sorgfailtige arbeit bildet ein hiibsches heftchen in der von professor Varnhagen herausgegebenen sehr achtenswerten sammlung der 'Erlanger Beitriige zur Englischen Philologie'. Auch den text der sechsten, der Edinburgher, hs. hat Kaufmann bei seinen untersuchungen benutzt. Da er jedoch der sehr fehlerhaften wiedergabe dieser letzten fassung in 'Turnbull's sammelbändchen 'The Visions of Tundale, elc.' und einer text- 